\title{
Effects of the beam loading in the rf deflectors of the CLIC test facility CTF3 combiner ring
}

\author{
David Alesini and Alessandro Gallo \\ INFN Laboratori Nazionali di Frascati, P.O. Box 13, I-00044, Frascati (Roma), Italy
}

(Received 19 November 2003; published 7 April 2004)

\begin{abstract}
In this paper we study the impact of the rf deflectors beam loading on the transverse beam dynamics of the CTF3 combiner ring. A general expression for the single-passage wake field is obtained. Different approximated formulas are derived applying linearization of the $\mathrm{rf}$ deflector dispersion curve either on a limited or an unlimited frequency range. A dedicated tracking code has been written to study the multibunch multiturn effects on the transverse beam dynamics. The numerical simulations reveal that the beam emittance growth due to the wake field in the rf deflectors is a small fraction of the design emittance if the trains are injected perfectly on axis. Nevertheless in case of injection errors the final emittance growth strongly depends on the betatron phase advance between the rf deflectors. If the finite bunch length is included in the tracking code, the scenario for the central part of the bunches does not change. However, for some particular injection errors, the tails of the bunches can increase the total transverse bunch emittances.
\end{abstract}

DOI: 10.1103/PhysRevSTAB.7.034403

PACS numbers: 29.27.Bd, 42.79.Fm, 29.27.Ac

\section{INTRODUCTION}

The CTF3 (CLIC test facility 3) [1] is the third test facility of the CLIC (Compact Linear Collider) project [2-4]. The CTF3 main goal is to prove the feasibility of the $30 \mathrm{GHz}$ rf power production in a long (140 ns) pulse by using the bunch recombination technique. The conceptual layout of CTF3 is shown in Fig. 1.

The frequency multiplication by the novel bunch interleaving technique is one of the most important issues to be tested. In CTF3 a long $(1.4 \mu \mathrm{s})$ train of $20 \mathrm{~cm}$ spaced short bunches (drive beam) is converted into a short (140 ns) train of $2 \mathrm{~cm}$ spaced bunches. In the first stage the pulse is compressed by a factor of 2 by using a transverse rf deflector at $1.499 \mathrm{GHz}$ and a $42 \mathrm{~m}$ circumference delay loop. An 84 m circumference combiner ring (CR) is used in the second stage to further compress the pulse by a factor of 5 [5]. This will be achieved by means of two rf deflectors working at $2.998 \mathrm{GHz}$ already constructed $[6,7]$ and successfully tested in the CTF3 preliminary phase [8].

In this paper we investigate the beam loading effects in the rf deflectors of the CR which may result in transverse bunch emittance growth, particle loss, and degradation of the drive beam quality thus reducing effectiveness of power conversion at $30 \mathrm{GHz}$. In Sec. II we analyze the single- and multipassage wake field models that we have introduced to study the multibunch effects. In Sec. III we illustrate the results obtained with the tracking code in the case of a perfect injection of the bunches or considering different injection errors. Finally, in Sec. IV, we illustrate the results obtained including, in the tracking code, a finite bunch length. The results are summarized in

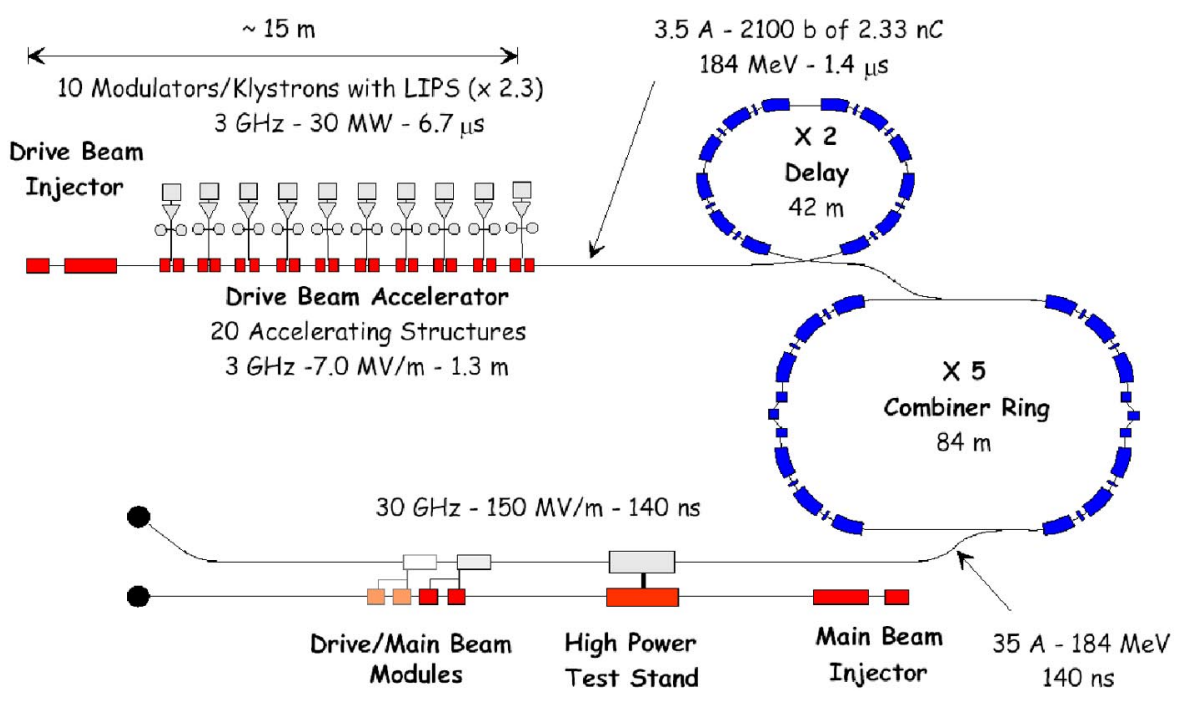

FIG. 1. (Color) Conceptual layout of CTF3. 
TABLE I. Main CTF3 parameters at the injection/extraction of the CR.

\begin{tabular}{llc}
\hline \hline & Energy $(E)$ & $180(\mathrm{MeV})$ \\
& Circumference length $(L)$ & $84 \mathrm{~m}$ \\
& Bunch charge $\left(Q_{b}\right)$ & $2.33(\mathrm{nC})$ \\
& Bunch length $\left(\sigma_{z}\right)$ & $1.5-2.5(\mathrm{~mm})$ \\
& Beam emittance ${ }^{\mathrm{a}}(\varepsilon)$ & $\sim 0.4(\mathrm{~mm} \mathrm{mrad})$ \\
& Number of bunches per pulse $\left(N_{b}\right)$ & 2100 \\
Injection & $\left\{\begin{array}{lc}\text { Pulse duration }\left(\tau_{\mathrm{CR}} \text { inj }\right. \\
\text { Number of trains per pulse }\left(N_{p \text { inj }}\right)\end{array}\right.$ & $1.36(\mu \mathrm{s})$ \\
Bunch separation $\left(\tau_{B}\right)$ & 5 \\
Extraction & $\left\{\begin{array}{l}\text { Pulse duration }\left(\tau_{\mathrm{CR}} \text { extr }\right. \\
\text { Number of trains per pulse }\left(N_{p \text { extr }}\right) \\
\text { Bunch separation }\left(\tau_{A}\right)\end{array}\right.$ & $140(\mathrm{~ns})$ \\
\hline \hline
\end{tabular}

${ }^{\mathrm{a}}$ The definition of beam emittance used in this evaluation is $\varepsilon=\sqrt{\left\langle x^{2}\right\rangle\left\langle x^{\prime 2}\right\rangle-\left\langle x x^{\prime}\right\rangle^{2}}$, where $x$, $x^{\prime}$ are the position and angle of particles in the transverse phase space and \langle\rangle is the average value over the bunch.

Sec. V. ${ }^{1}$ The main parameters of CTF3 CR at the injection are reported in Table I.

\section{SINGLE- AND MULTIPASSAGE WAKE FIELD MODELS FOR RF DEFLECTORS}

The process of bunch train compression in the $\mathrm{CR}$ is illustrated in Fig. 2 and widely discussed in [1]. The efficiency required for the $\mathrm{rf}$ deflectors can be easily met by scaling already existing traveling wave (TW) or standing wave structures. On the contrary, the most demanding issues are those related to the beam dynamics, including the beam loading effects on the fundamental deflecting mode.

Strong beam loading effects can, in fact, affect both the beam transverse dimensions and the beam transport and, as a consequence, the efficiency of the power conversion at $30 \mathrm{GHz}$.

From this point of view it has been chosen to build TW rf deflectors since wake fields can leave the structure faster due to the lower filling time [6].
A couple of TW rf deflectors (Fig. 3) has already been constructed and tested in the CTF3 preliminary phase $[7,8]$. The main $\mathrm{rf}$ deflector parameters are reported in Table II.

\section{A. Single-passage wake models}

In order to study the effect of beam loading in $\mathrm{rf}$ deflectors on the transverse beam dynamics of CTF3 $\mathrm{CR}$, we have proposed a single-passage wake field model that has been implemented into a tracking code to analyze the multipassage effects. Below we give the model description.

The constructed rf deflectors are disk loaded backward waveguides (Lengeler-like structures [9]) working on the so-called hybrid mode $\mathrm{EH}_{11}$.

Referring to Fig. 4, the electromagnetic (e.m.) field around the axis of the deflector, in the case of small pitch approximation $(\lambda \gg D)$, negligible iris thickness $(t / D \ll 1)$, and phase velocity equal to $c$ is given by the equations [10]:

$$
\begin{aligned}
& \underline{E}=\left\{\begin{array}{c}
e_{r}=j \frac{E}{4}\left(k^{2} a^{2}+k^{2} r^{2}\right) \cos (\vartheta) \\
e_{\vartheta}=-j \frac{E}{4}\left(k^{2} a^{2}-k^{2} r^{2}\right) \sin (\vartheta) \\
e_{z}=E k r \cos (\vartheta)
\end{array}\right\} e^{j\left[\omega^{*}(t-z / c)+\phi\right]}=\underline{e}(r, \vartheta) \cdot e^{j\left[\omega^{*}(t-z / c)+\phi\right]}, \\
& \underline{H}=\left\{\begin{array}{c}
Z_{0} h_{r}=j \frac{E}{4}\left(k^{2} a^{2}-k^{2} r^{2}-4\right) \sin (\vartheta) \\
Z_{0} h_{\vartheta}=j \frac{E}{4}\left(k^{2} a^{2}+k^{2} r^{2}-4\right) \cos (\vartheta) \\
Z_{0} h_{z}=-E k r \sin (\vartheta)
\end{array}\right\} e^{j\left[\omega^{*}(t-z / c)+\phi\right]}=\underline{h}(r, \vartheta) \cdot e^{j\left[\omega^{*}(t-z / c)+\phi\right]},
\end{aligned}
$$

where $\omega^{*}$ is the working frequency, $k=\omega^{*} / c$, and $Z_{0}=$ $\sqrt{\mu_{0} / \varepsilon_{0}}$.

Using the expression of the Lorentz force acting on a particle of charge $q$ that moves along the structure on the plane $\vartheta=0$, with a velocity equal to $c$, one obtains

\footnotetext{
${ }^{1}$ The effects of the beam loading in the $1.499 \mathrm{GHz}$ rf deflector of the delay loop are not discussed here since this seems to be a less relevant issue.
}

$$
F_{T}=q \operatorname{Re}\left(E_{r}-Z_{0} H_{\vartheta}\right)=-q E \sin (\phi) .
$$

To evaluate the beam loading in the structure one has to consider both the interaction between the traveling charges and the transverse electric field $E_{r}$ (beam loading in phase) and between the traveling charges and the longitudinal electric field $E_{z}$ (beam loading $90^{\circ}$ out of phase). 


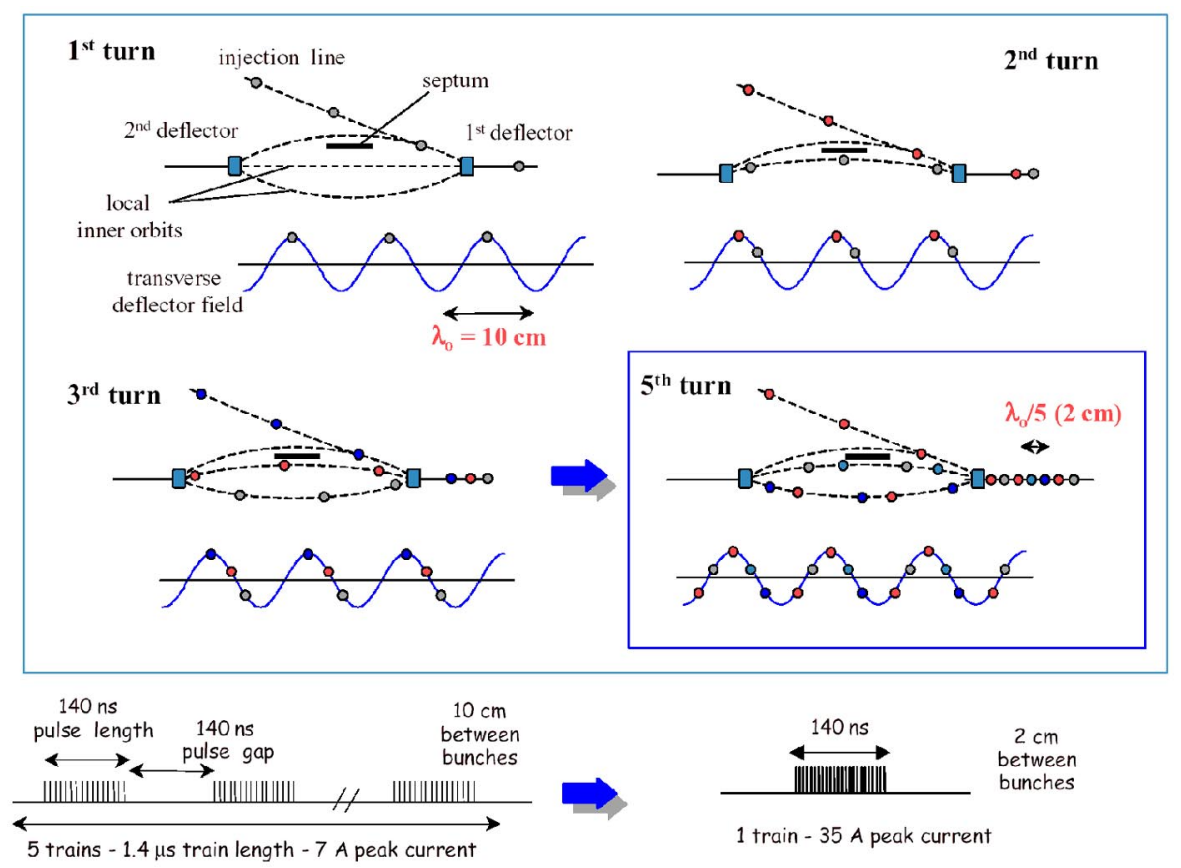

FIG. 2. (Color) Sketch of the bunch frequency multiplication in the CTF3 CR.

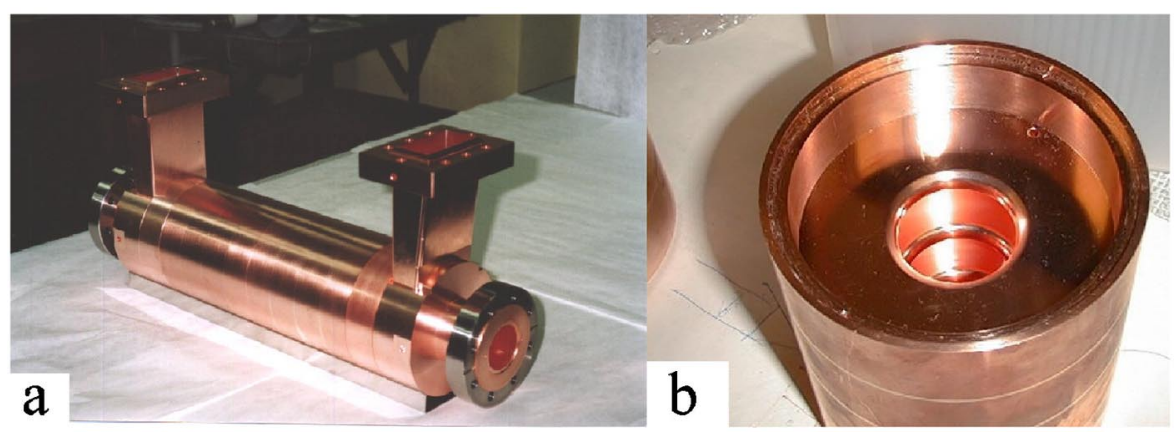

FIG. 3. (Color) (a) Constructed TW rf deflector for the CR; (b) detail of the iris loaded structure.

TABLE II. Combiner ring rf deflector parameters.

Frequency $(f)$

Cell length $(D)$

Cell radius $(b)$

Iris internal radius $(a)$

Iris thickness $(t)$

Number of active cells $\left(N_{c}\right)$

Phase advance per cell $(\beta D)$

Deflector length $(L)$

Group velocity $\left(v_{g} / c\right)$

Filling time $\left(\tau_{F}\right)$

Shunt impedance per unit length $\left(r_{s}\right)$

$r_{s} / Q$

Input power $\left(P_{\text {in }}\right)$

Deflection $\left(\phi_{D}\right)$
$2.99855(\mathrm{GHz})$

$33.33(\mathrm{~mm})$

$56.01(\mathrm{~mm})$

$21.43(\mathrm{~mm})$

$9.53(\mathrm{~mm})$

10

$2 \pi / 3$

$33(\mathrm{~cm})$

$-0.0237$

$47(\mathrm{~ns})$

$18.1(\mathrm{M} \Omega / \mathrm{m})$

$1425 \Omega / \mathrm{m}$

$2 \mathrm{MW}$

5 (mrad)

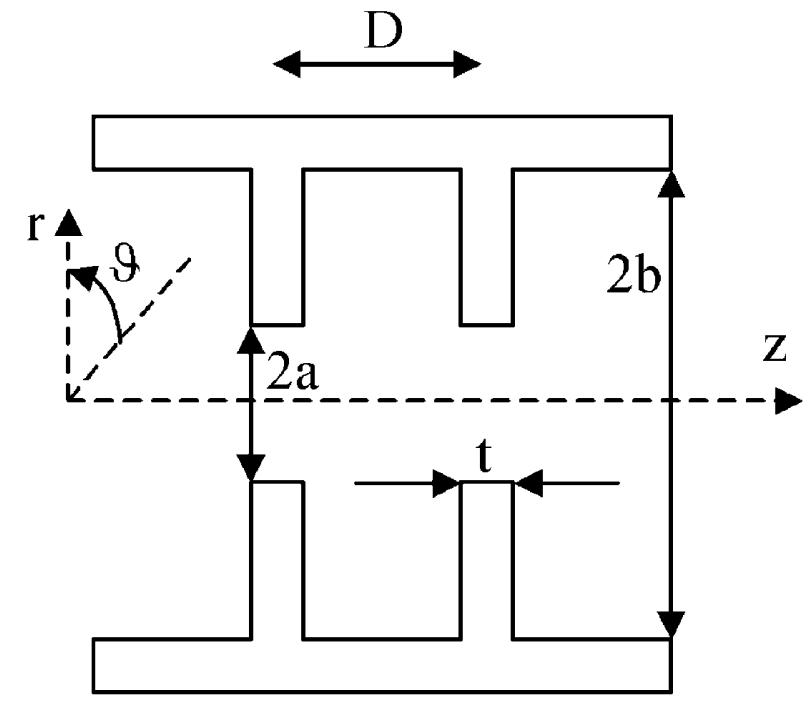

FIG. 4. Sketch of the disk loaded rf deflector structure. 
The first contribution is similar to the standard beam loading in a LINAC accelerating section with the exception that, in this case, is the transverse component of the bunch velocity that couples with the $E H_{11} E$ field. The deflection spread along the train can be estimated obtaining a quite small value in the CTF3 case [11].

The second contribution is of more concern because in the CR the bunch pattern is such that, at a certain time, the deflector will be crossed by bunch trains off axis and with a phase separation of $2 \pi / 5$ generating a mutual perturbation mainly through the out of phase wake.

In order to calculate the wake field generated by the interaction between the traveling charges and the longitudinal electric field one has to refer to the general problem of modes excitation by an electric current $J$ that flows through a waveguide [12].

The deflecting field excited by the passage of an ultrarelativistic particle of charge $q$ (Fig. 5) can be written in the frequency domain as

$$
\underline{E}^{+}=c^{+}(\omega, z) \underline{e}(\omega, r, \vartheta) .
$$

Considering only the interaction between the longitudinal component of the current and the deflecting mode, the coefficient $c^{+}$that gives the amplitude of the excited field, is given by the equation (see Appendix A):

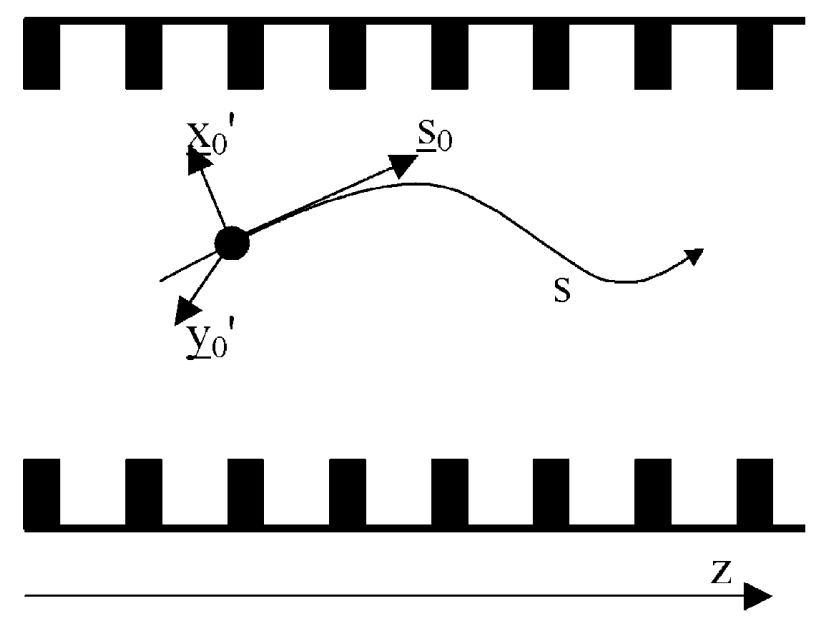

FIG. 5. Sketch of a charge $q$ moving inside the rf deflector.

$c^{+}(\omega, z)=\frac{q}{4 \Pi(\omega)} \int_{z}^{L} e_{z}\left[\omega,\left.\underline{r}\left(z^{\prime}\right)\right|_{\text {part.traj. }}\right] e^{-j \omega\left(z^{\prime} / c\right)} e^{j \beta(\omega) z^{\prime}} d z^{\prime}$,

where $\left.\underline{r}\left(z^{\prime}\right)\right|_{\text {part.traj. }}$ is the transverse position of the particle along the structure, $\beta$ is the propagation constant of the TW deflecting mode, and $\Pi$ is the power flow along the structure (negative in the case of backward wave).

The e.m. field of the excited mode can be calculated, in time domain, by the Fourier integral:

$$
\begin{aligned}
& \underline{E}^{+}(t, z, r, \vartheta)=\frac{1}{2 \pi} \int_{-\infty}^{+\infty} c^{+}(\omega, z) \underline{e}_{t}(\omega, r, \vartheta) e^{-j \beta(\omega) z} e^{j \omega t} d \omega=\frac{1}{\pi} \operatorname{Re}\left[\int_{0}^{+\infty} c^{+} \cdot \underline{e}_{t} \cdot e^{-j \beta z} e^{j \omega t} d \omega\right], \\
& \underline{H}^{+}(t, z, r, \vartheta)=\frac{1}{2 \pi} \int_{-\infty}^{+\infty} c^{+}(\omega, z) \underline{h}_{t}(\omega, r, \vartheta) e^{-j \beta(\omega) z} e^{j \omega t} d \omega=\frac{1}{\pi} \operatorname{Re}\left[\int_{0}^{+\infty} c^{+} \cdot \underline{h}_{t} \cdot e^{-j \beta z} e^{j \omega t} d \omega\right] .
\end{aligned}
$$

In order to calculate the coefficient $c^{+}$and the corresponding e.m. field for all $t$ and $z$, one has to use Eqs. (4) and (5) making a double integration in the variables $z^{\prime}$ and $\omega$. Unfortunately, these expressions are very time consuming for applications in multibunch multiturn tracking codes. To find a simplified expression for the wake field calculation, some approximations have to be done.

\section{Approximation 1: linearization of the dispersion curve in a limited range of frequency}

The typical dispersion curve of an rf deflector is plotted in Fig. $6, f^{*}$ being the working frequency, i.e., the frequency to a phase velocity $\left(v_{\mathrm{ph}}\right)$ equal to $c$.
Taking into account the expressions (4) and (5), it is easy to show that the major contribution, in time domain, to the deflecting force acting on a trailing particle $90^{\circ}$ out of phase from the leading one ${ }^{2}$, comes from a small range of frequencies near $f^{*}$. For such a particle the real part of the coefficient $c^{+}$has a local maximum for $f=f^{*}$ because the exponential term oscillates for $f \neq f^{*}$ and the deflecting force has a maximum when the particle and the wave are synchronous.

It is possible, therefore, to linearize the previous expressions near the point $\left(\beta^{*}, f^{*}\right)$ obtaining the following expressions for the coefficient $c^{+}$(Appendix B):

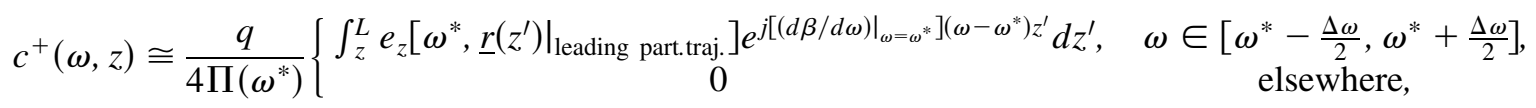

where $\left[\omega^{*}-\Delta \omega / 2, \omega^{*}+\Delta \omega / 2\right]$ is an interval centered around the frequency $f^{*}$.

The expression for the electric field in the time domain becomes

\footnotetext{
${ }^{2}$ It means that the particle samples the wake field at time $t=$ $T / 4+h T+z / c h=0,1,2, \ldots$, where $T=1 / f^{*}$ is the period of the wave in the deflector.
} 


$$
\underline{E}^{+}(t, z)=\frac{q}{2 \pi \Pi\left(\omega^{*}\right)} \operatorname{Re}\left[\underline{e}\left(\omega^{*}\right) e^{j \omega^{*}(t-z / c)}\right] \int_{z}^{L} e_{z}\left[\omega^{*},\left.\underline{r}\left(z^{\prime}\right)\right|_{\text {leading part.traj. }}\right] \frac{\Delta \omega}{2} \operatorname{sinc}\left[\left(t^{*}-\frac{z-z^{\prime}}{v_{g}}\right) \frac{\Delta \omega}{2}\right] d z^{\prime},
$$

where $\operatorname{sinc}(x)=\sin (x) / x$.

The Lorentz force acting on the trailing particle moving on the plane $\vartheta=0$ is given by

$$
F_{T}\left(t^{*}, z\right)=q\left(E_{r}-Z_{0} H_{\vartheta}\right.
$$

where $t^{*}=T / 4+h T h=0,1,2, \ldots$

\section{Approximation 2: linearization of the dispersion curve over an unlimited range of frequency}

This is the case of Eq. (8) when $\Delta \omega \rightarrow \infty$ obtaining $^{3}$

$$
\begin{gathered}
\underline{E}^{+}(t, z)=\frac{q\left|v_{g}\right|}{2 \Pi\left(\omega^{*}\right)} \operatorname{Re}\left[\underline{e}\left(\omega^{*}\right) e^{j \omega^{*}(t-z / c)}\right] e_{z}\left[\omega^{*},\left.\underline{r}\left(z-t v_{g}\right)\right|_{\text {leading part.traj. translated in } z=-t v_{g}}\right] \\
F_{T}\left(t^{*}, z\right) \cong-\frac{q^{2}\left|v_{g}\right|}{2 \Pi\left(\omega^{*}\right)} E \sin \left(\omega^{*} t^{*}\right) e_{z}\left[\omega^{*},\left.\underline{r}\left(z-t^{*} v_{g}\right)\right|_{\text {leading part.traj. translated in } z=-t v_{g}}\right] .
\end{gathered}
$$

Introducing the $R / Q$ of the structure [9] given by the formula:

$$
\frac{R}{Q}=\frac{E_{\perp}^{2} / P}{Q}=\frac{E^{2} v_{g}}{c \Pi\left(\omega^{*}\right) k}
$$

where $E_{\perp}$ is the equivalent maximum deflecting field, $P$ is the dissipated power per unit length, and $Q$ is the quality factor per unit length, one obtains the following field expression:

$$
\underline{E}^{+}=-\frac{1}{2} q \omega^{*} \frac{R}{Q} k r\left(z-t v_{g}\right) \operatorname{Re}\left[\frac{\underline{e}\left(\omega^{*}\right)}{E} e^{j \omega^{*}(t-z / c)}\right]
$$

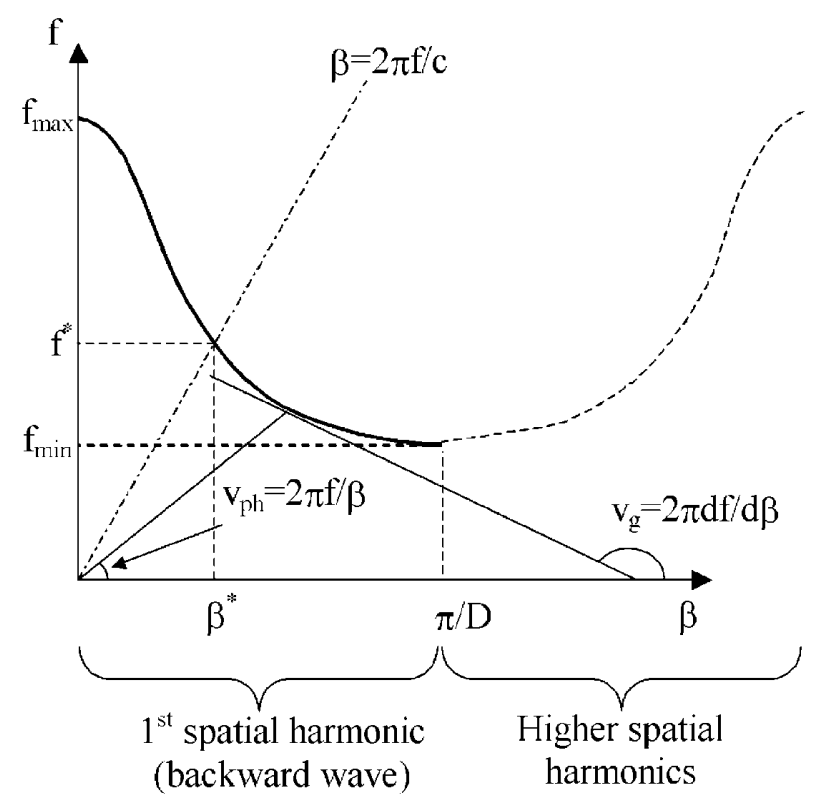

FIG. 6. Sketch of a typical dispersion curve for a TW rf deflector. and for the transverse force the expression:

$$
F_{T}\left(t^{*}, z\right)=\frac{1}{2} q^{2} \omega^{*} \frac{R}{Q} k r\left(z-t^{*} v_{g}\right) \sin \left(\omega^{*} t^{*}\right) .
$$

These expressions for the wake field and force reproduce the most intuitive behavior of a field generated by the passage of a particle in a generic waveguide (see Appendix C): an envelope of the field (or force) that follows the profile of the leading particle trajectory (this is because the field $E_{z}$ is proportional to the displacement $r$ of the particle from the axis of the structure) rigidly translating along the structure with a negative group velocity equal to $v_{g}$ with a positive phase velocity equal to $c$.

\section{Comparison between the different expressions for the wake field}

With the rf deflector dimensions of Table II, one obtains, with the analytical formulas given in [10], the dispersion curve plotted in Fig. 7.

It is possible to evaluate the transverse field excited by a leading charge and probed by a trailing particle injected $90^{\circ}$ out of phase with respect to the leading one by using the most accurate formula (5) or the simplified ones (8)-(13).

Let us consider, as an example, a parabolic leading particle trajectory (that corresponds to the trajectory of a particle injected in phase with the deflecting field and not affected by wake field effects) of the type:

$$
\left.\underline{r}(z)\right|_{\text {leading part.traj. }}=r_{\text {in }}+r_{\text {in }}^{\prime} z+\frac{1}{2} \frac{\Delta r^{\prime}}{L} z^{2},
$$

where $r_{\text {in }}$ and $r_{\text {in }}^{\prime}$ are the initial injection position and

\footnotetext{
${ }^{3}$ Remembering that $\lim _{\Delta \omega \rightarrow \infty}(\Delta \omega / 2) \operatorname{sinc}\left\{\left[t-\left(z-z^{\prime} / v_{g}\right)\right] \times\right.$ $(\Delta \omega / 2)\}=\pi\left|v_{g}\right| \delta\left[z^{\prime}-\left(z-t v_{g}\right)\right]$.
} 


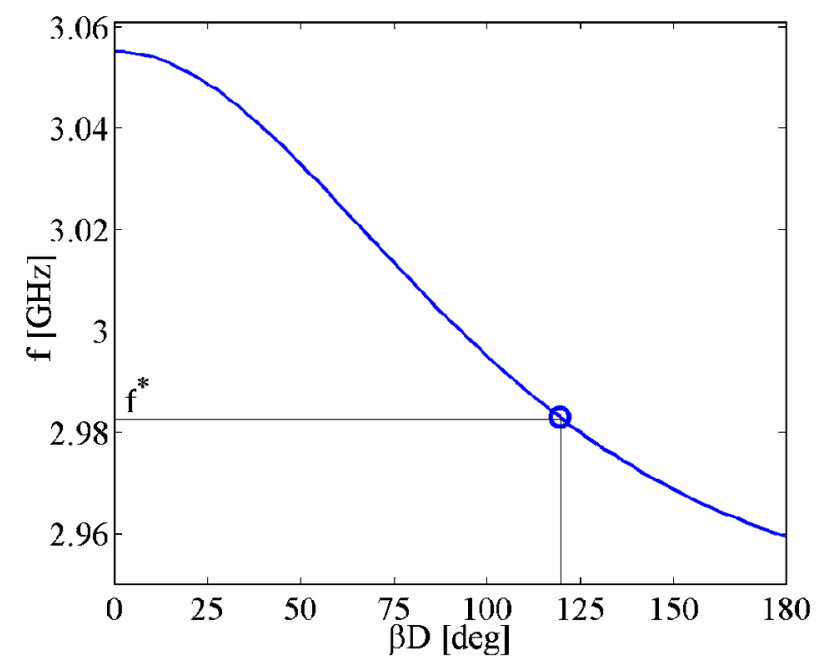

FIG. 7. (Color) Dispersion curve of the rf deflector obtained analytically.

angle and $\Delta r^{\prime}$ is the deflection angle inside the deflector. Three different possible trajectories are plotted in Fig. 8. The obtained transverse forces ${ }^{4}$ probed by a particle that enters the structure after a time $t_{1}^{*}=T / 4$ (solid line) and $t_{2}^{*}=T / 4+80 \mathrm{~T}$ (dashed line) from the leading one are plotted in Fig. 9(a) in the case of trajectory 1 of Fig. 8. The correct result obtained by the numerical integration of Eq. (5) is compared with those obtained in the linear approximation of the dispersion curve in the passband interval of the $E H_{11}$ mode $\left[\omega_{1}, \omega_{2}\right]$ and with those obtained by the linear approximation of the dispersion curve in an unlimited range of frequencies. The same quantities, for trajectories 2 and 3 of Fig. 8, are plotted in Figs. 9(b) and 9(c).

Introducing the transverse wake probed by a trailing particle that enters the structure after a time $t_{n}^{*}=T / 4+$ $n T$ defined as

$$
w_{\perp n}=\frac{1}{q^{2}} \int_{0}^{L} F_{T}\left(t_{n}^{*}, z\right) d z
$$

one obtains the results plotted in Figs. 10(a)-10(c) for the three different trajectories, respectively.

\section{B. Multipassage wake fields}

In the case of an infinite train of bunches spaced in time by $T$ and with the same trajectory inside the deflector, the density current is given by

\footnotetext{
${ }^{4}$ The force is calculated on the axis of the structure. More precisely, as shown in Eqs. (8) and (13), in the approximated cases the transverse force does not depend on the displacement of the trailing particle. Nevertheless, considering the exact field distribution and the correct calculation of Eqs. (2)-(5), there is a force dependence due to the transverse position of the trailing particle.
}
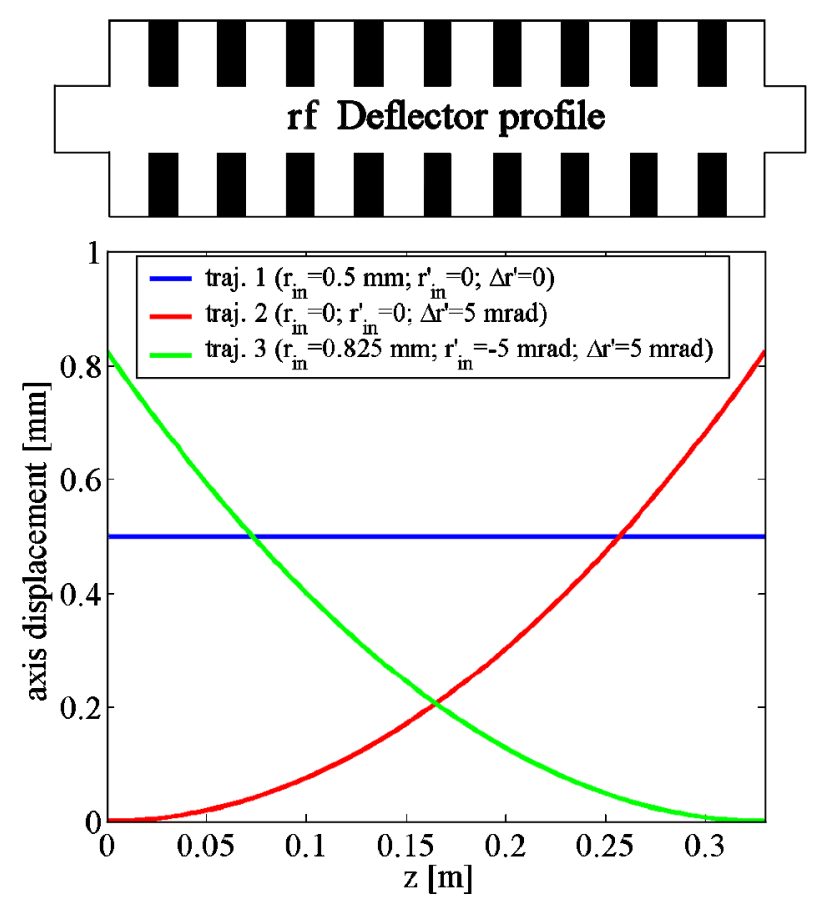

FIG. 8. (Color) Considered trajectories of the leading particle.

$$
\underline{J}(t)=q \underline{s}_{0}(s) \sum_{i=-\infty}^{\infty} \delta\left(t-\frac{s}{c}+i T\right) \delta\left(x^{\prime}\right) \delta\left(y^{\prime}\right)
$$

that, in the frequency domain, becomes

$$
\underline{J}(\omega)=q \underline{s}_{0}(s) \delta\left(x^{\prime}\right) \delta\left(y^{\prime}\right) \omega^{*} e^{-j \omega(s / c)} \sum_{n=-\infty}^{\infty} \delta\left(\omega-n \omega^{*}\right),
$$

where $\omega^{*}=2 \pi / T$.

In order to evaluate the coefficient $c^{+}$one has to remember that the CTF3 rf deflector frequency is $2.998 \mathrm{GHz}$ and that the dispersion curve for the mode $E H_{11}$, for these structures, has a passband of the order of a few hundred MHz. Furthermore, the trains of bunches have spectrum lines separated by the distance $(1 / T)$, that, during the recombination process, is bigger or equal to $2.998 \mathrm{GHz}$. For this reason the coefficients $c^{+}$, in the case of a train of bunches, can be simply obtained substituting the term $e^{-j \omega z / c}$ in Eq. (4) with the spectrum (17), obtaining, for parabolic trajectories:

$$
c^{+}(\omega, z)=\frac{q \omega^{*} E k}{4 \Pi\left(\omega^{*}\right)} \delta\left(\omega-\omega^{*}\right) c(z)
$$

where

$$
c(z)=r_{\text {in }}(L-z)+\frac{1}{2} r_{\text {in }}^{\prime}\left(L^{2}-z^{2}\right)+\frac{1}{6} \frac{\Delta r^{\prime}}{L}\left(L^{3}-z^{3}\right) .
$$




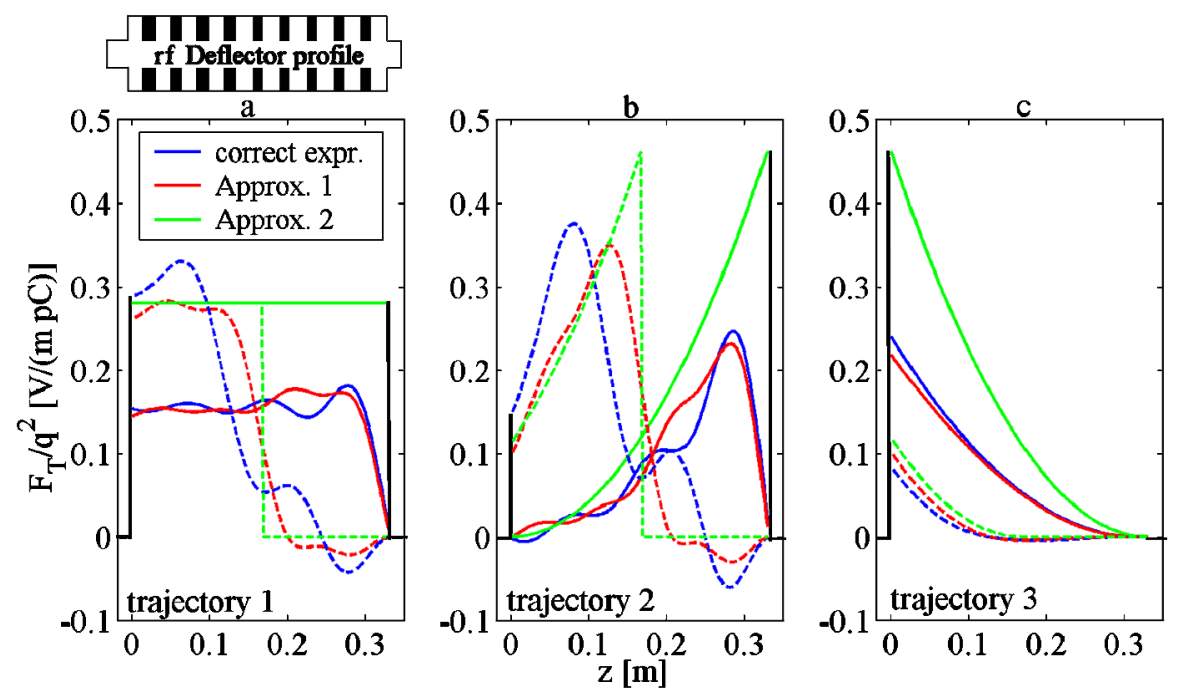

FIG. 9. (Color) Transverse force probed by a trailing particle.
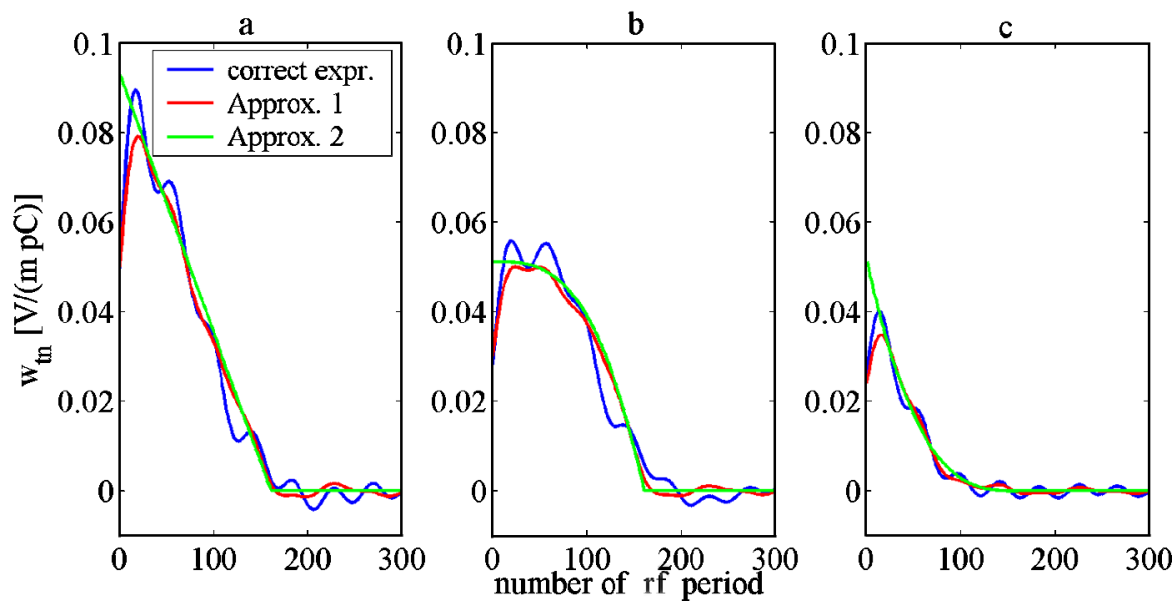

FIG. 10. (Color) Transverse wake probed by a trailing particle that enters in the structure after a time $t_{n}^{*}=T / 4+n T(n=$ number of $\mathrm{rf}$ period).

In this case the electric field is given by

$$
\underline{E}^{+}(t, z)=\frac{q \omega^{*} E k}{4 \pi \Pi\left(\omega^{*}\right)} c(z) \operatorname{Re}\left[\underline{e}\left(\omega^{*}\right) e^{j \omega^{*}(t-z / c)}\right],
$$

and the transverse force seen by a trailing particle of charge $q$ that enters the deflector at the time $t^{*}$ is given by

$$
F_{T}\left(t^{*}, z\right)=-\frac{q^{2} \omega^{*} k E^{2}}{4 \pi \Pi\left(\omega^{*}\right)} \sin \left(\omega^{*} t^{*}\right) c(z) .
$$

The obtained results are plotted in Figs. 11(a)-11(c) for a trailing particle that enter the structure out of phase with respect to the particles of the infinite train. In this case the correct solution does not differ from the case of a linearized dispersion curve in a limited range of frequencies (approximation 1). In fact the beam spectrum, given by (17), samples the two curves in the point $\omega=\omega^{*}$ only and the corresponding wake field is the same. The case of linear approximation over an unlimited range of frequencies (solid line) differs slightly with respect to the two previous cases (dashed line).

\section{TRACKING CODE RESULTS}

The three different models for the wake field produce regime multibunch forces along the deflector that are almost identical even if, in the single-passage case, the force probed by the particle is different in the three cases. The explanation is that the multibunch regime solution is the response to a "monochromatic" excitation and that the details of the dispersion curve out of resonance are not relevant in this case.

Moreover, the integrated force (wake), also in the single passage, is very similar in the three cases.

For these reasons, the model of the single-passage wake fields that has been adopted to study the multibunch 


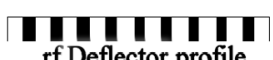

rf Deflector profile
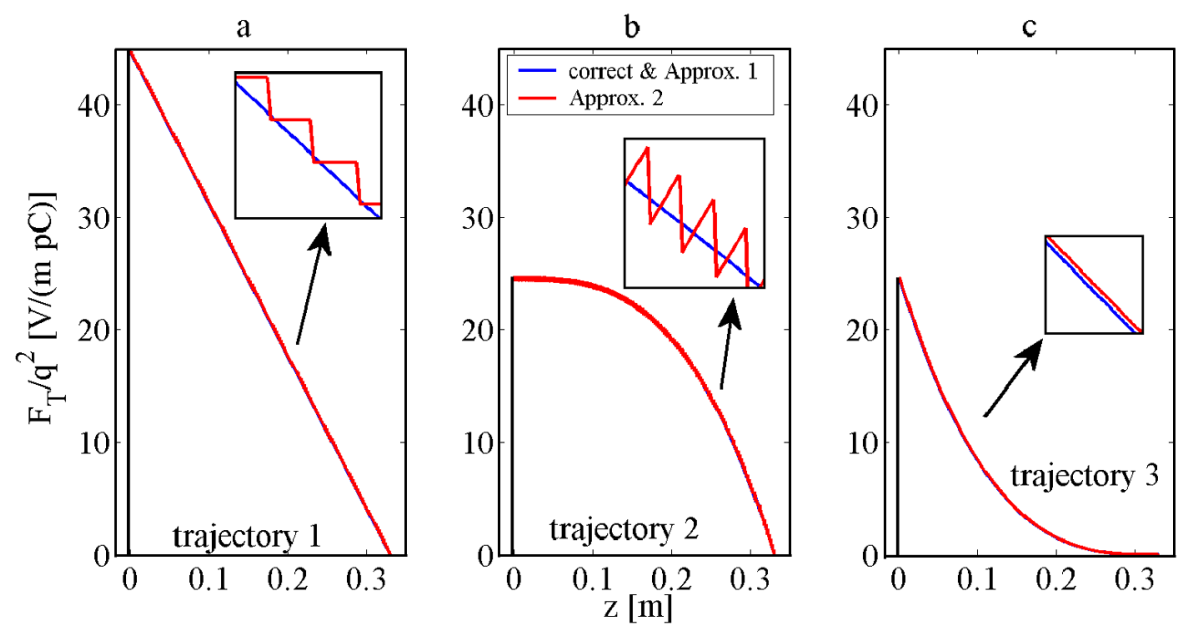

FIG. 11. (Color) Transverse force seen by a $90^{\circ}$ out of phase particle in the case of a multibunch regime.

regime with a tracking program, is the simplest one (approximation 2).

The tracking code scheme is sketched in Fig. 12. Each bunch, represented as a macroparticle, enters the 1st deflector with some horizontal initial conditions $\left(x_{\text {in }}, x_{\text {in }}^{\prime}\right)$, interacts with both the main rf deflecting field and the wake left by the bunches ahead, contributes to the wake, and exits from the deflector with some new horizontal conditions $\left(x_{\text {out }}, x_{\text {out }}^{\prime}\right)$. The bunch, then, is transported to the other deflector by the transport matrix $M_{21}$, interacts with the rf field and wakes of this second device, and so on.

At the end of the merging process (five trains and five turns) each macroparticle ends up with certain horizontal conditions $\left(x_{\mathrm{out}}, x_{\mathrm{out}}^{\prime}\right)$ corresponding to a certain value of the Courant-Snyder invariant $I_{\text {out }}$ associated with the motion of the bunch center of mass.

The tracking allows studying, therefore, the distribution of the final values of the Courant-Snyder invariants for all bunches and its dependence on the deflector wakes, injection errors, ring optical function, and phase advance (first evaluations of these effects are reported in [13]).

\section{A. Perfect injection of the five trains}

This is the case of bunch trains injected with the initial conditions that perfectly match the main deflecting field of the deflectors so that, if there were no wake, all the bunches would end up on the combiner ring design orbit. This condition corresponds to the point $x_{\mathrm{in}}=0.825 \mathrm{~mm}$ and $x_{\mathrm{in}}^{\prime}=-5 \mathrm{mrad}$ of the phase space at the injection plane.

The 1st train makes the first revolution alone, and there are no bunches interacting with its out of phase wake. During the second revolution, there is the contemporary presence of the 1st and 2nd trains, which cross the deflectors with some horizontal displacement. The two trains are interleaved with a separation of $2 \pi / 5$ of $\mathrm{rf}$ period and their bunches interact through the out of phase wake. This generates a first perturbation that deviates the

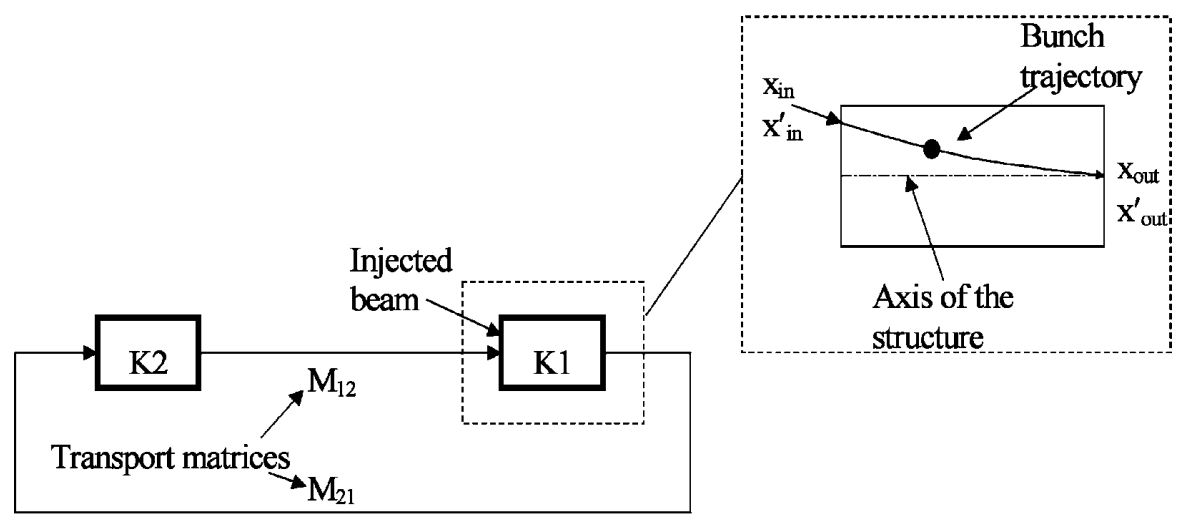

FIG. 12. Tracking code scheme. 

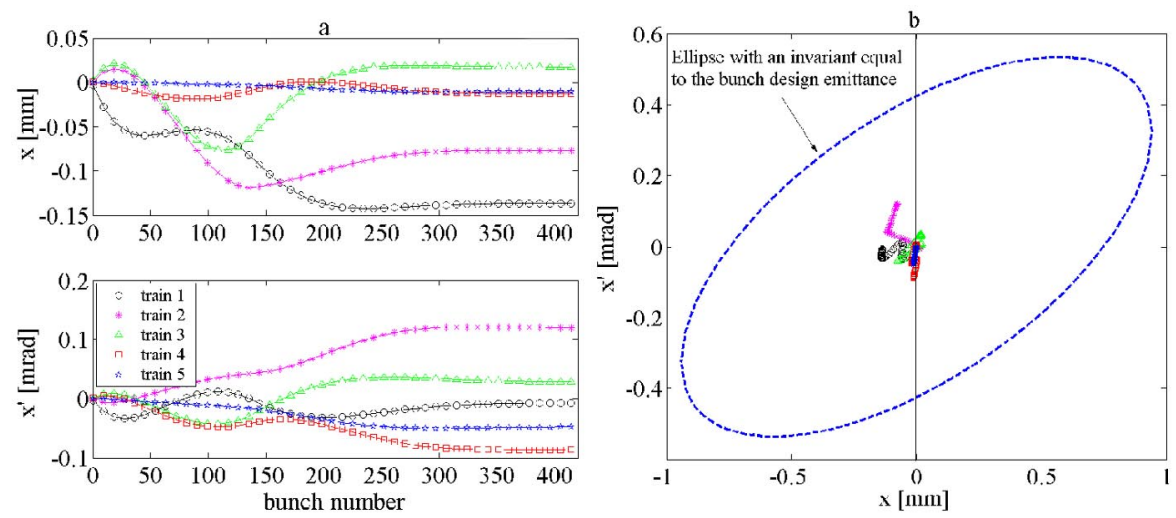

FIG. 13. (Color) Positions and angles of the bunches with respect to the nominal orbit taken at the 1st deflector output at the end of the merging process (a). Corresponding plot in the phase space (b).

bunches from their ideal trajectories. Similar processes take place during the next interleaving phases and, when the five trains are finally merged in a single one, the bunches are spread by a certain extent in the horizontal phase space.

A plot of the position and angle of the bunches with respect to the nominal orbit taken at the 1st deflector output at the end of the merging process is shown in Fig. 13(a). The corresponding plot in the phase space is shown in Fig. 13(b). To compare the final bunch distributions in the phase space with the horizontal beam emittance, in the plot it is also reported the ellipse with a Courant-Snyder invariant equal to the horizontal beam emittance. The Courant-Snyder invariants of bunches are shown in Fig. 14. In the tracking code the transport matrices $M_{12}$ and $M_{21}$ are given by the nominal combiner ring optics [1].

Figures 13 and 14 represent the so-called "systematic effect" of the wake field since the perturbations in the final bunch transverse positions are not driven by initial

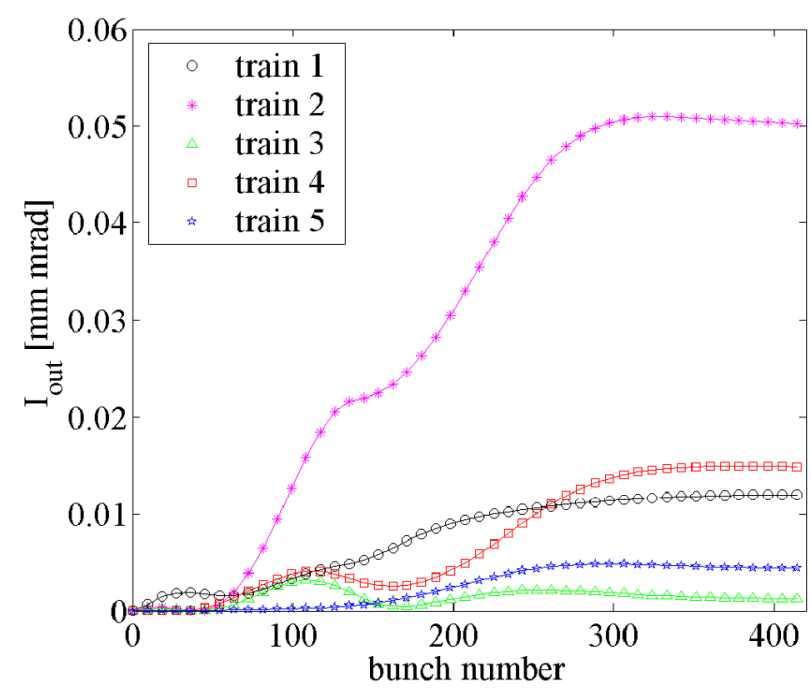

FIG. 14. (Color) Courant-Snyder invariant of the bunches after the recombination process. injection errors. The average and maximum values of the Courant-Snyder invariants are, respectively, $I_{\mathrm{av}} \cong$ $1 \times 10^{-2} \mathrm{~mm}$ mrad, $\quad I_{\max } \cong 5 \times 10^{-2} \mathrm{~mm} \mathrm{mrad}$. The spread of the macroparticle Courant-Snyder invariant values caused by the systematic effect is a small fraction of the CTF3 bunch design emittance $(\varepsilon \cong$ $0.4 \mathrm{~mm} \operatorname{mrad} @ 180 \mathrm{MeV}$ ) and, in principle, does not cause problems in the bunches distribution after the merging process.

\section{B. Errors in the injection of trains}

Referring to the case of an equal injection error for all the bunches of the five incoming trains $s^{5}$, the buildup mechanism for the final invariant spread is just the same as that described previously even if, in this case, the initial errors can drive the process to larger final errors.

The bunch train footprints in the horizontal phase space at the output of the 1st deflector for an injection offset of $1 \mathrm{~mm}$ and for an injection error of $0.633 \mathrm{mrad}$ in angle (both corresponding to an initial value of the Courant-Snyder invariant $I_{\text {in }}=0.716 \mathrm{~mm} \mathrm{mrad}$ ) are reported in Figs. 15(a) and 15(b). As done in the previous paragraph, in the plots it is also reported the ellipse with a Courant-Snyder invariant equal to the horizontal beam emittance. The corresponding Courant-Snyder invariants of bunches are reported in Figs. 16(a) and 16(b).

By performing different simulations with different initial conditions it may be seen that, in general, the maximum and average values of $I_{\text {out }}$ are not constant for a given initial value $I_{\text {in }}$ of the invariant, but, due to the nature of the wake, depend also on the position and angle errors of the incoming trains. Different injection errors (Fig. 17), corresponding to a given $I_{\text {in }}$, give different maximum and average invariant values of the final distributions, as shown in Fig. 18 ( $\left.I_{\text {in }}=0.716 \mathrm{~mm} \mathrm{mrad}\right)$.

\footnotetext{
${ }^{5}$ This is the case, for instance, if one assumes that the error can fluctuate only from pulse to pulse due to some jitter in the beam transport.
} 

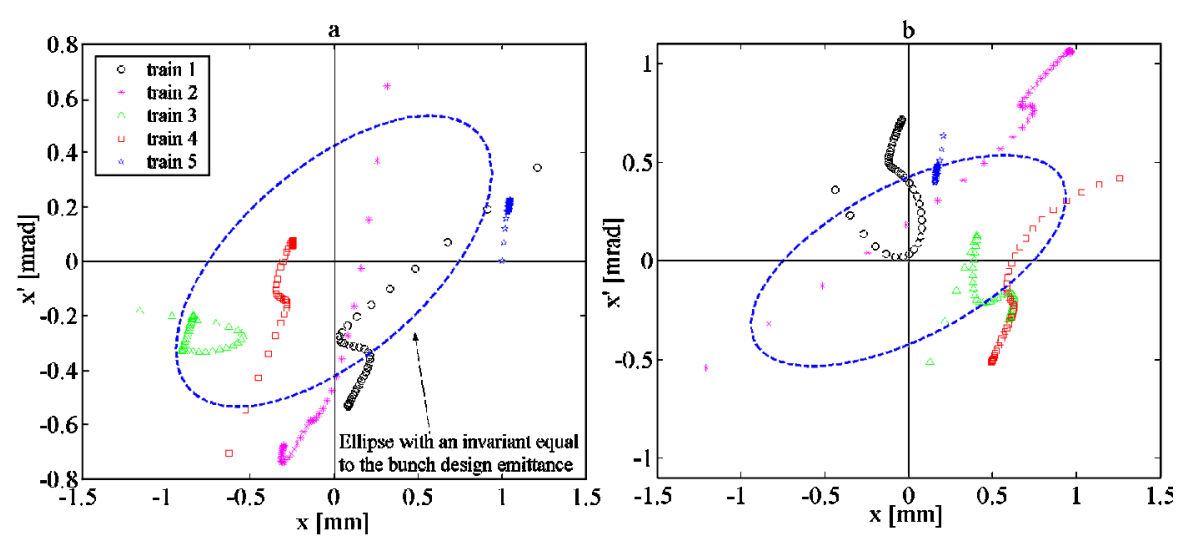

FIG. 15. (Color) Bunch train in the horizontal phase space for an injection offset of $1 \mathrm{~mm}$ (a) and for an injection error of $0.633 \mathrm{mrad}$ in angle (b).
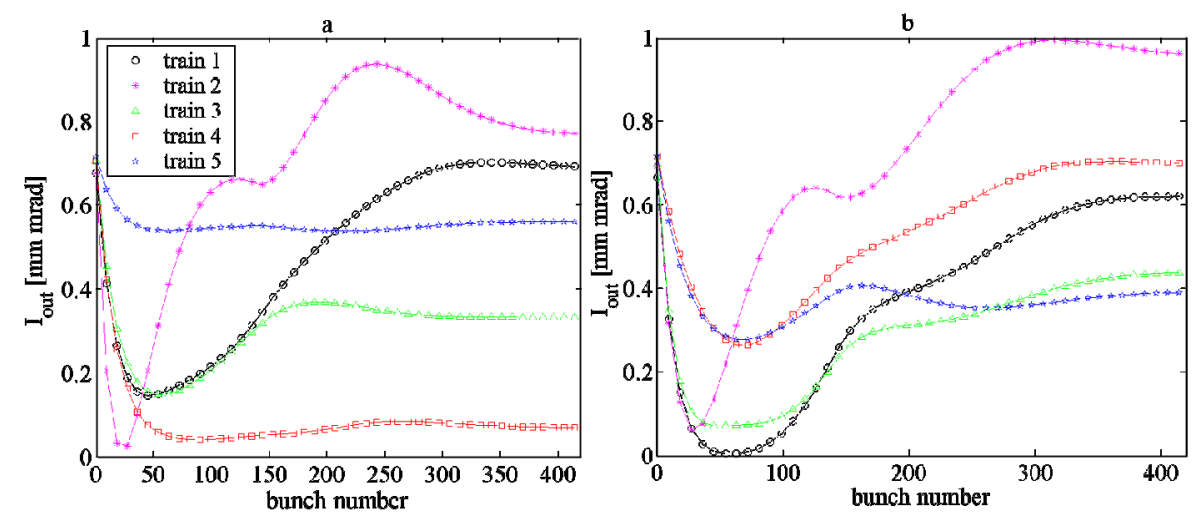

FIG. 16. (Color) Courant-Snyder invariant distributions for an injection offset of $1 \mathrm{~mm}$ (a) and for an injection error of $0.633 \mathrm{mrad}$ in angle (b).

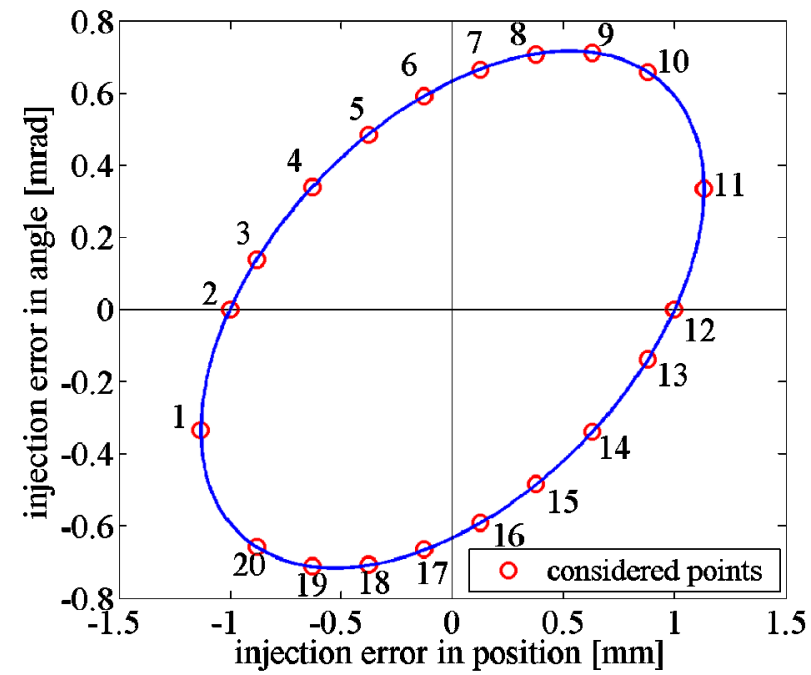

FIG. 17. (Color) Possible injection errors in the horizontal input phase space.

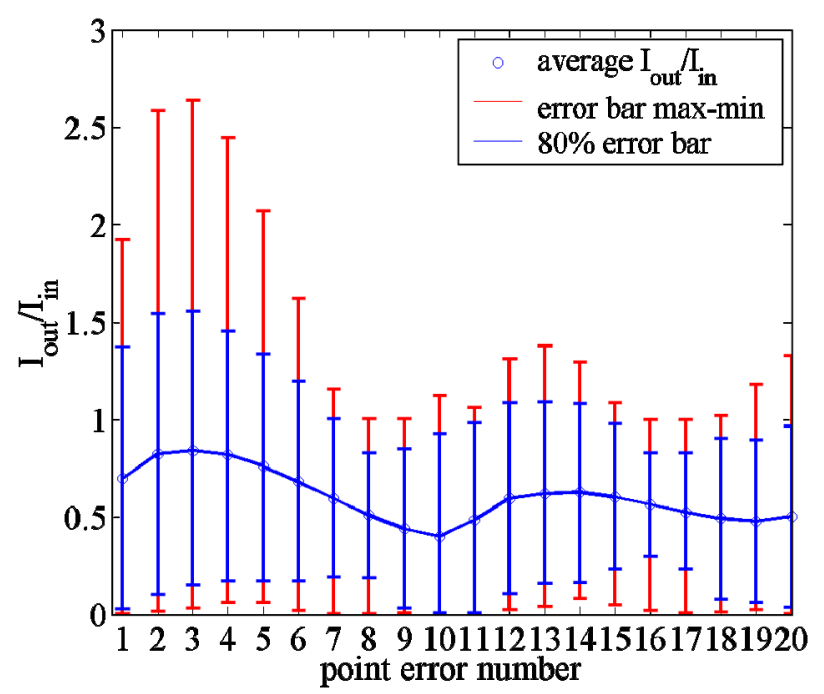

FIG. 18. (Color) Average invariant with the relative error bars for any possible injection error $\left(I_{\mathrm{in}}=0.716 \mathrm{~mm} \mathrm{mrad}\right)$. 


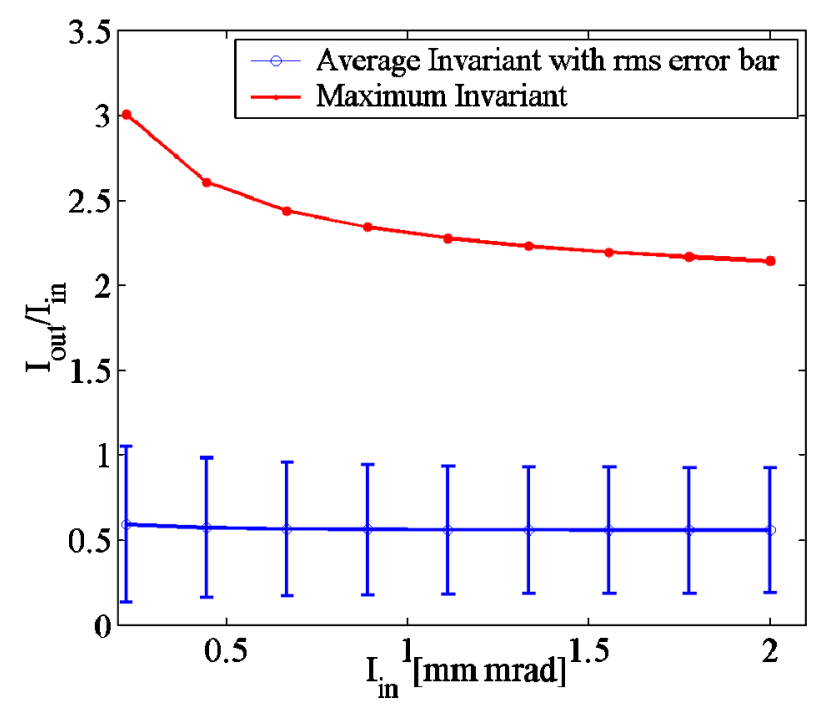

FIG. 19. (Color) Maximum and average values of the final invariant as a function of the input invariant.

For any given initial value $I_{\text {in }}$, there is an error that gives the largest final invariants value $\left(I_{\text {out max }}\right)$ and the largest average invariant value ( $\left.I_{\text {out av }}\right)$.

The plots of $I_{\text {out max }}$ and $I_{\text {out av }}$ (this last including the \pm rms error bar) for $I_{\text {in }}$ ranging from 0.2 to $2 \mathrm{~mm} \mathrm{mrad}$ are shown in Fig. 19.

It may be seen that, for the optics parameters considered and with the exception of the $I_{\text {in }} \cong 0$ case, it is always $I_{\text {out av }}<I_{\text {in }}$, which means that, on the average, the deflectors wake gives a sort of "cooling" of the Courant-Snyder invariant of the bunch center of mass. On the other hand, from the same figure one has $I_{\text {out } \max } / I_{\text {in }}<3$ and $I_{\text {out }} / I_{\text {in }}<1$ for all bunches staying within 1 standard deviation above the average $(\approx 70 \%$ of the bunches).

The previous results have been obtained considering the nominal betatron phase advance [1] between deflectors 1 and 2. Different phase advances give different amplification factors and the minimization of the ratios $I_{\text {out }} / I_{\text {in }}$ is one of the constraints in the CR lattice design.

As an example, the ratios $I_{\text {out }} / I_{\text {in }}$, for various values of the betatron phase advance, are reported in Figs. 20 for the case of an injection error caused by a pure displacement of $1 \mathrm{~mm}$. At the nominal phase advance of $262^{\circ}$ this ratio is close to the minimum. Modifications of the phase advance in the range of $\pm 10^{\circ}$ do not significantly change the scenario. Anyway, some tunes outside that range show magnification factors larger than 100.

When the betatron phase advance from $K 1$ to $K 2$ is around $270^{\circ}$ there is a sort of cooling of the initial Courant-Snyder invariants while there is a strong increase of the initial invariant in the case of betatron phase advances around $90^{\circ}$. The mechanism can be understood looking at the injection of the first two trains and considering, for simplicity, a recombination factor 4 , i.e., a second train injected exactly $1 / 4$ of wavelength ahead of the first train. Let us consider the case of $270^{\circ}$ phase advance and a positive displacement error at injection (for instance $+1 \mathrm{~mm}$, as reported in Fig. 20). We have the following:

(a) Train 1 in deflector 1: the bunches of the first train are injected $+1 \mathrm{~mm}$ of axis in deflector 1 ; they generate a pure out of phase wake that does not interact with the bunches of the first train itself [Eq. (13)].

(b) Train 1 in deflector 2: since the phase advance of the ring is $270^{\circ}$ the bunches enter in deflector 2 with a positive angle and, to the first order, they generate a negligible wake (we can neglect for simplicity the trajectory inside the deflector and consider it with a negligible length).

(c) Trains 1 and 2 in deflector 1: the bunches of train 2 are injected $+1 \mathrm{~mm}$ of axis in deflector 1 ahead of the corresponding bunches of train 1, while the bunches of train 1 come back to deflector 1 with zero displacement
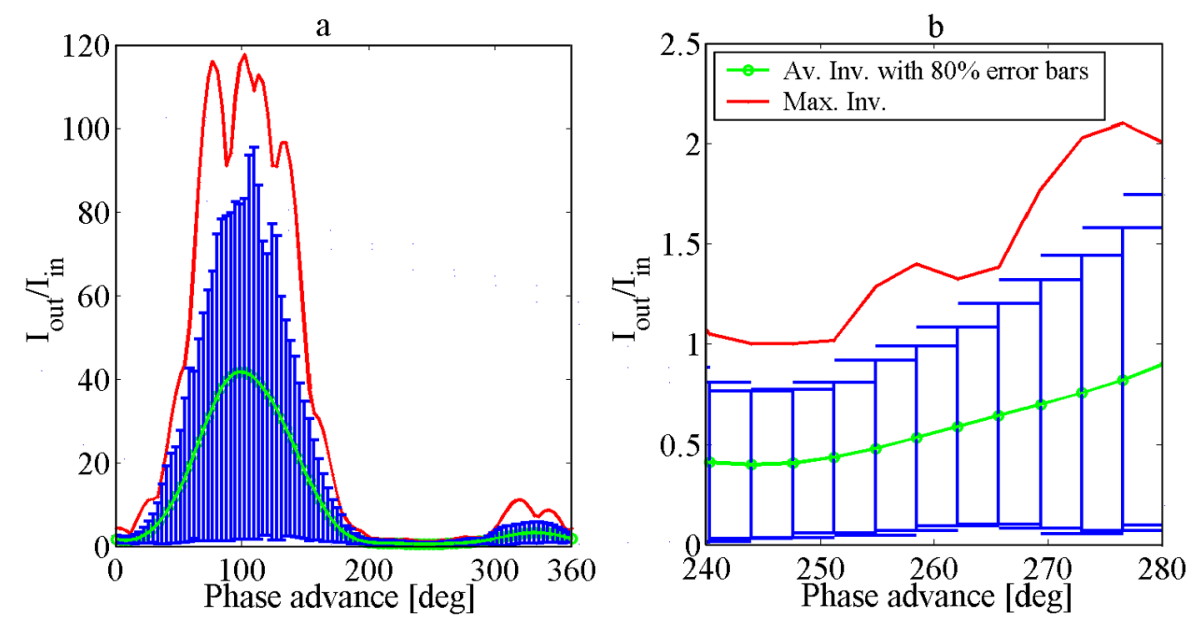

FIG. 20. (Color) Ratios $I_{\mathrm{omax}} / I_{\mathrm{in}}$ and $I_{\mathrm{oav}} / I_{\mathrm{in}}$ in the case of an injection error caused by a pure displacement of $1 \mathrm{~mm}$ for various values of the betatron phase advance. 
$1^{\text {st }}$ Deflector - Injection of train 2

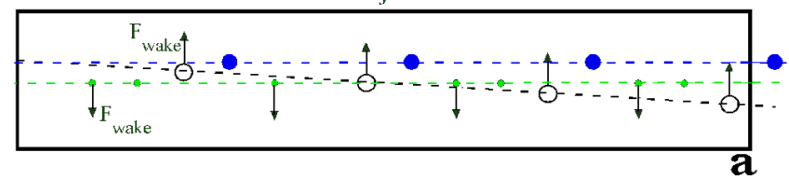

$2^{\text {nd }}$ Deflector $-1^{\text {st }}$ passage of train 1 and 2 together

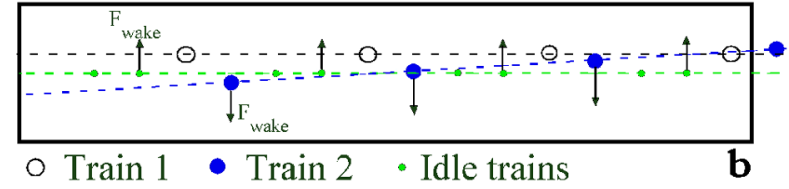

FIG. 21. (Color) Sketch of trains 1 and 2 passing through deflectors 1 and 2 to explain the damping mechanism at $270^{\circ}$ of the ring phase advance.

and a negative angle (remember that the phase advance of one turn is $270^{\circ}+180^{\circ}=90^{\circ}$, and a positive displacement is transformed in a negative angle after one turn). Therefore the bunches of train 2 generate a pure out of phase wake kicking positively the bunches of train 1 [see Eq. (13)] and partially compensating their negative angle [Fig. 21(a)]. On the contrary, to the first order, the bunches of train 1 generate a negligible out of phase wake.

(d) Trains 1 and 2 in deflector 2: the bunches of train 2 enter in the deflector with a positive angle while the bunches of train 1 enter with a positive displacement. Therefore the bunches of train 1 generate a pure out of phase wake kicking negatively the bunches of train 2 [see Eq. (13) again] partially compensating their negative angle [Fig. 21(b)]. The bunches of train 2 generate, to the first order, a negligible out of phase wake.

This mechanism repeats at each turn also for the other trains and gives the cooling of the initial invariants. Using the same arguments, it is easy to demonstrate that a phase advance of $90^{\circ}$ gives an increase of the initial error. It is important to remark that this result is completely inverted if the bunches are injected in the ring behind the circulating ones, as shown also from simulations.

\section{EFFECT OF A FINITE BUNCH LENGTH}

The design bunch length of CTF3 is between 1.5 and $2.5 \mathrm{~mm} \mathrm{[4],} \mathrm{and} \mathrm{it} \mathrm{is} \mathrm{a} \mathrm{very} \mathrm{important} \mathrm{parameter} \mathrm{to} \mathrm{be}$ controlled in order to have a good efficiency in the power generation, since the spectrum of longer bunches rolls off below $30 \mathrm{GHz}$. Furthermore, as illustrated in the following, an increase of the bunch length can give loss of current due to the fact that the tails of the bunch may grow in terms of Courant-Snyder invariant because of the finite wave length of the rf deflectors field and wakes. On the other hand an increase of the bunch length, reducing the charge density, may give, at high bunch current, a reduction of the collective effect in terms of coherent synchrotron radiation and interaction with the short range wake field [4].

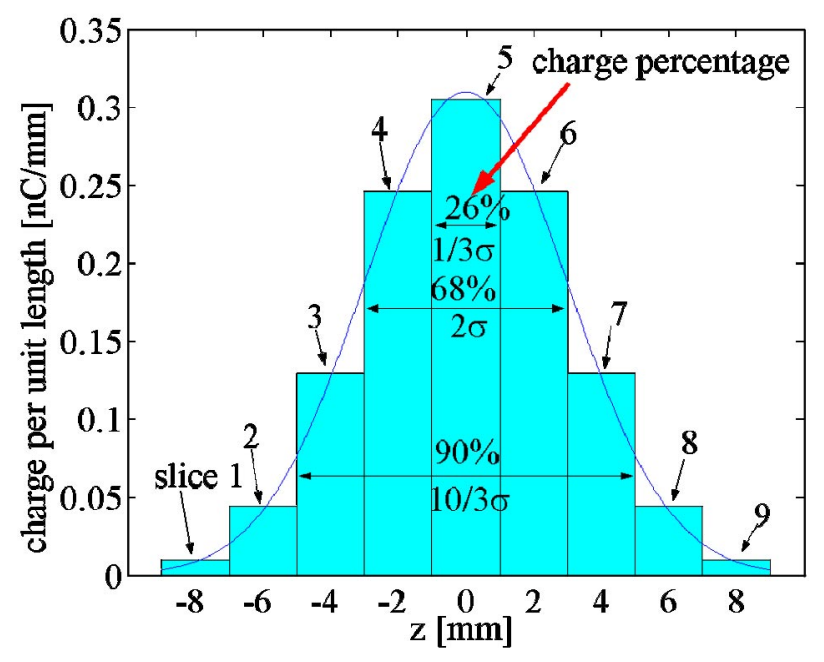

FIG. 22. (Color) Discretization of the bunch in a finite number of slices.

The value of $\sigma_{z}=3 \mathrm{~mm}$ seems to be a maximum acceptable value for the bunch length.

The effects of the beam loading in the rf deflectors considering a finite bunch length can be simulated dividing the bunches into slices (Fig. 22). Each slice can be considered as a macroparticle and the complete simulation can be performed.

First, assuming a perfect injection of the trains and the absence of the wake field in the deflectors, one obtains the transverse bunch slices output positions after the recombination plotted in Figs. 23(a) and 23(b). As seen there is an increase of the rms bunch emittance calculated with respect to the central slice ${ }^{6}$ of the bunch that is a small fraction of the bunch design emittance (Table III). This is due to the lower kick given to the tails with respect to the central slice.

The effect of the beam loading is shown in Figs. 24(a) and 24(b). In this case it has been considered the nominal phase advance of the ring and a perfect injection of the trains. In Fig. 24(b) the output invariants of the central slices of bunches are reported as a function of the bunch number. Comparing this figure with Fig. 14 one can observe that, for the central slice of bunches, the output invariants are comparable with those obtained without considering the bunch length.

The rms emittances with respect to the central slice are reported in Fig. 25. In this case, also, the increases of the emittance are a small fraction of the bunch design emittance.

\footnotetext{
${ }^{6}$ The definition of rms beam emittance with respect to the central slice is rms emitt $=\sqrt{\left\langle x^{2}\right\rangle\left\langle x^{\prime 2}\right\rangle-\left\langle x x^{\prime}\right\rangle^{2}}$, where $x, x^{\prime}$ are the position and angle of the slices in the transverse phase space with respect to the central slice, and \langle\rangle is the average value over the bunch weighed with the slice charge.
} 

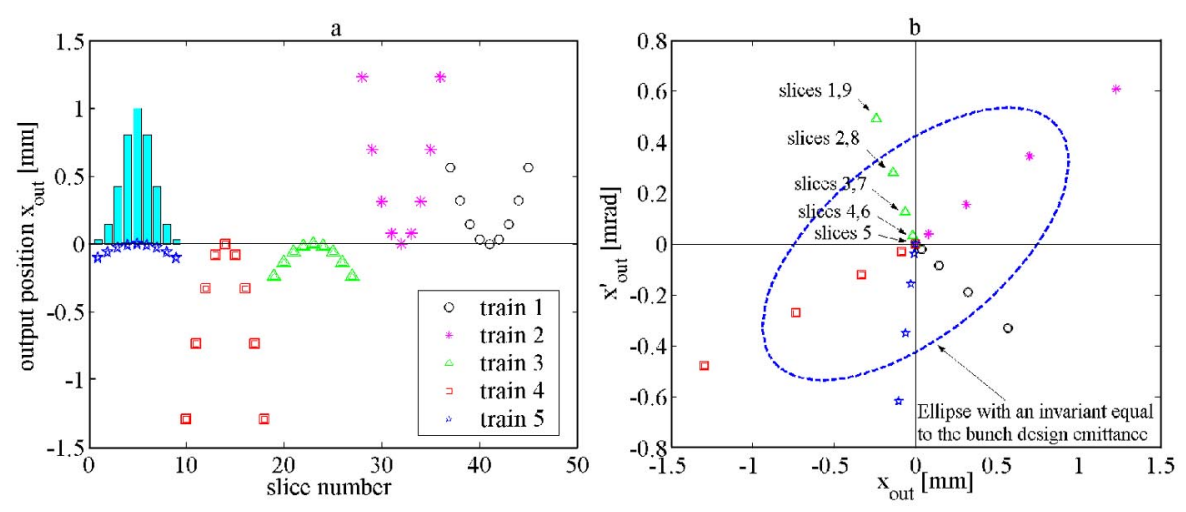

FIG. 23. (Color) (a) Output positions of the bunch slices in the case of perfect injection of the trains and the absence of the wake field; (b) slices in the phase space.

TABLE III. rms emittance of bunches referred to the central slice in the case of perfect injection of the trains and absence of the wake field

\begin{tabular}{cr}
\hline \hline $\begin{array}{c}\text { Train } \\
\text { No. }\end{array}$ & $\begin{array}{r}\text { rms emittance } \\
(\mathrm{mm} \mathrm{mrad})\end{array}$ \\
\hline 1 & $1.38 \times 10^{-4}$ \\
2 & $7.73 \times 10^{-5}$ \\
3 & $4 \times 10^{-5}$ \\
4 & $1.86 \times 10^{-5}$ \\
5 & $1.13 \times 10^{-6}$ \\
\hline \hline
\end{tabular}

The output invariants of the central slices and the rms emittances have been calculated in the case of different injection errors. The results are plotted in Figs. 26(a) and 26(b). The beam loading effects do not change, for the central slices of bunches, the scenario discussed in the case of $\sigma_{z}=0$. Concerning the other slices, we observed that the rms emittance of each bunch is, in the worst case, of the order of $15 \%$ of the value of the Courant-Snyder invariant of the central slice. To explain this we can observe that, for a pure out of phase wake, the field

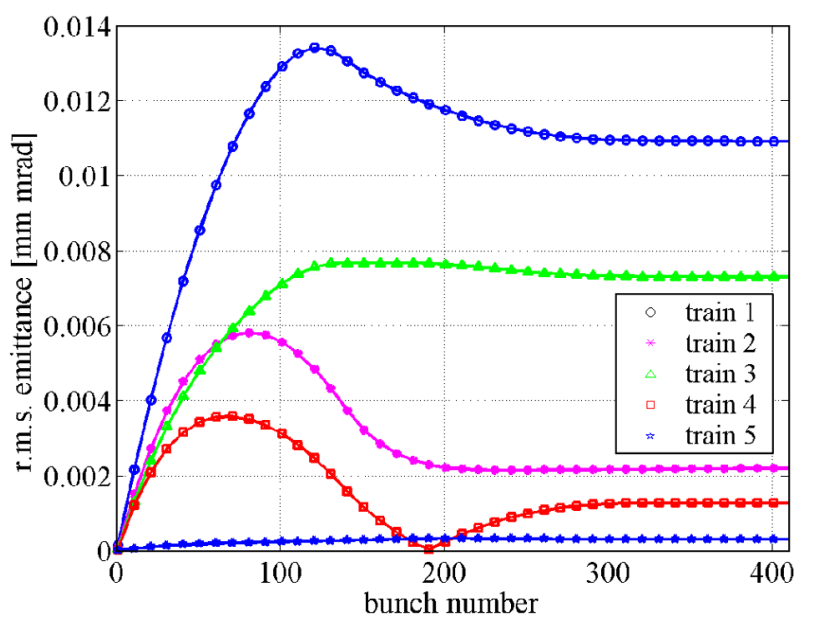

FIG. 25. (Color) rms emittances of bunches with respect to the central slice.

generated by a certain train does not interact with the central slices of the bunches of the same train because they are positioned at the wake zero crossing [see Eq. (13)]. On the contrary the tails of bunches are displaced from the zero crossing and receive a kick
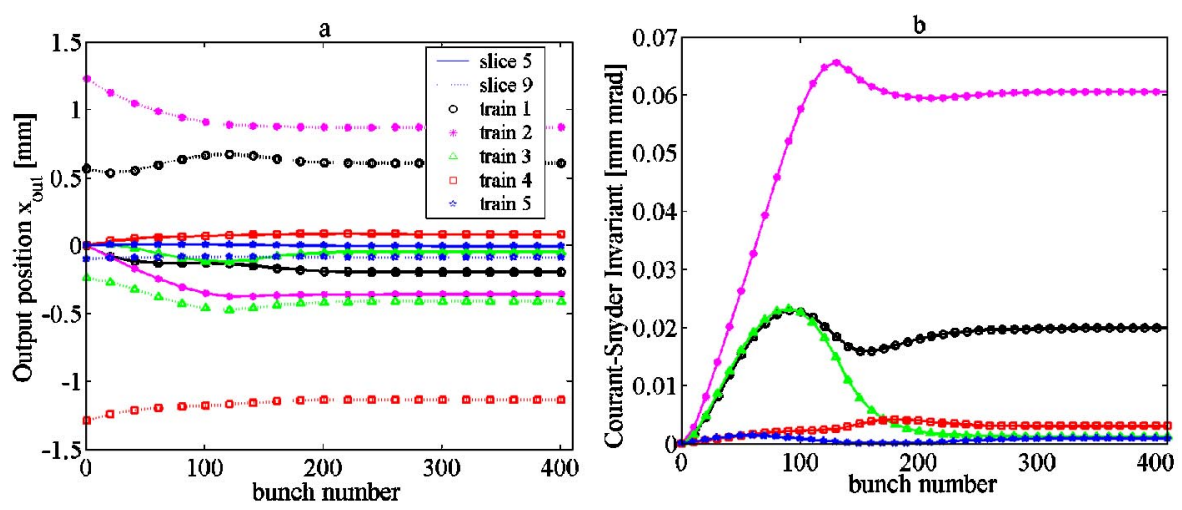

FIG. 24. (Color) (a) Output position of the slices as a function of the bunch number without injection errors; (b) output invariant of the central slices of bunches. 

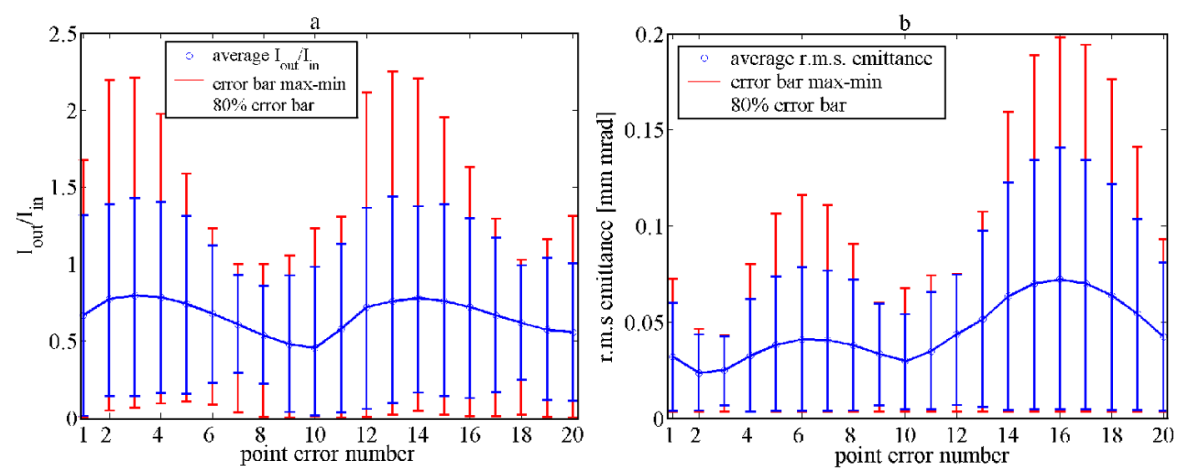

FIG. 26. (Color) (a) Output invariants for the central slices of bunches for any possible injection error; (b) rms emittances with respect to the central slice.

proportional to the displacement. Since at $3 \mathrm{GHz}$ the wavelength is $10 \mathrm{~cm}$ and the tails of a $3 \mathrm{~mm}$ rms bunch extend between $\pm 9 \mathrm{~mm}$ (Fig. 22), the emittance of the slices in the tails may significantly grow. The rms emittance growth due to the finite bunch length is, in the worst case, a factor of 2 lower than the bunch design emittance (for the considered injection errors) and can be considered a controllable effect.

\section{CONCLUSIONS}

In this paper we have studied the impact of beam loading in the rf deflectors on the transverse beam dynamics in the CTF3 combiner ring. For this purpose:

(1) We have obtained a general expression for the single-passage wake field. It has been shown that the general expression can be simplified by applying a linearization of the rf deflector dispersion curve over either a limited or an unlimited frequency range. Comparison of three wake field models has shown that the simplest wake field formula, obtained in the case of linearization over an unlimited frequency range, can be safely applied to describe multibunch multiturn wake field effects.

(1) A dedicated tracking code based on the simplest wake model has been written to study the transverse beam dynamics of bunch trains in the CTF3 CR. The numerical simulations have revealed that:

(a) the beam emittance growth due to the wake field in the rf deflectors is negligible if the trains are injected perfectly on axis;

(b) in case of injection errors the final emittance growth depends strongly on the betatron phase advance between the rf deflectors. For the worst phase advance values the emittance magnification factor may exceed 100. Instead, for the nominal phase advance of $260^{\circ}$ the emittance growth is close to its minimum;

(c) simulations taking into account the finite bunch length show that the scenario does not change for the central part of the bunches with respect to the case of zero bunch length. However, for some particular injection errors, the bunch tails can contribute to the increase of the total transverse bunch emittances.

As a general remark, the effects of the beam loading in the rf deflectors on the beam transverse phase space are considered tolerable, as long as the betatron phase advance between the kickers is close to the optimum value $\left(260^{\circ} \pm 10^{\circ}\right)$, the injection position and angle are not too large $\left(\Delta x_{\text {in }} \leq 1 \mathrm{~mm}, \Delta x_{\text {in }}^{\prime} \leq 0.7 \mathrm{mrad}\right)$, and the bunches are short enough $\left(\sigma_{z} \leq 3 \mathrm{~mm}\right)$.

\section{ACKNOWLEDGMENTS}

The authors would like to thank R. Corsini and D. Schulte for discussions on the subject and to have developed a first tracking code that has been of fundamental importance to validate, compare, and check our simulation results. Thanks also to M. Zobov for helpful suggestions and to A. Ghigo, responsible for the CTF3 collaboration at LNF.

\section{APPENDIX A: CALCULATION OF THE AMPLITUDE COEFFICIENT $c^{+}$OF THE EXCITED FIELD [EQ. (4)]}

Considering a set of independent modes (characterized with the subscript $n$ ), the general propagating field in a structure can be written, in frequency domain, as [12] (the subscripts $t$ and $z$ indicate the transverse and the longitudinal components of the field)

$$
\begin{aligned}
& \underline{E}^{ \pm}=\sum_{n=0}^{N} c_{n}^{ \pm} \underline{e}_{n} e^{-j\left( \pm \beta_{n}\right) z}=\sum_{n=0}^{N} c_{n}^{ \pm}\left(\underline{e}_{t n} \pm e_{z n} \underline{z}_{0}\right) e^{-j\left( \pm \beta_{n}\right) z}, \\
& \underline{H}^{ \pm}=\sum_{n=0}^{N} c_{n}^{ \pm} \underline{h}_{n} e^{-j\left( \pm \beta_{n}\right) z}=\sum_{n=0}^{N} c_{n}^{ \pm}\left( \pm \underline{h}_{t n}+h_{z n} \underline{z}_{0}\right) e^{-j\left( \pm \beta_{n}\right) z},
\end{aligned}
$$

where the signs " \pm " refer to the case of positive or negative phase velocities, respectively, $N$ is the number of excited modes, $c_{n}^{ \pm}$and $\beta_{n}$ are the amplitudes and the propagation constants of the $n$th mode, and $\underline{h}_{n}, \underline{e}_{n}$ are the magnetic and electric field distributions of the $n$th mode. 


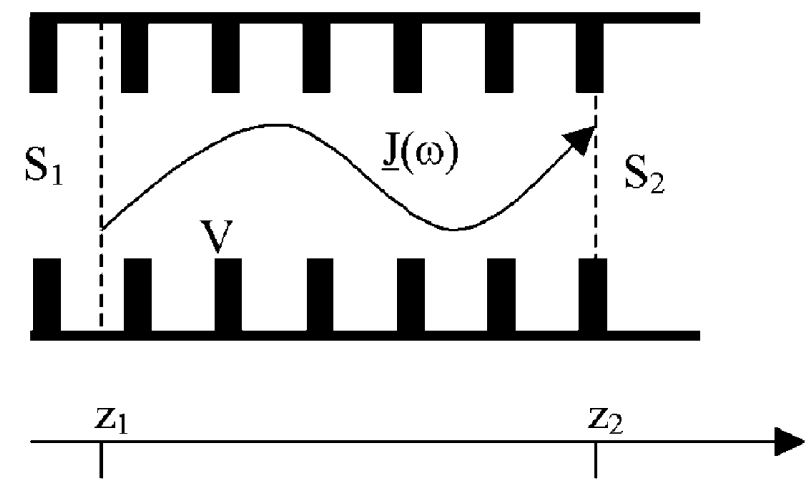

FIG. 27. Sketch of the deflecting structure excited by an electric current.

Considering an electric density current $J(\omega)$ in the structure (Fig. 27), it is possible to calculate the general coefficients $c_{n}^{+}$of the Eq. (A1) by the simple formula [12]:

$$
\begin{gathered}
c_{n}^{+}(\omega)=\mp \frac{\int_{V}\left(\underline{e}_{t n}-e_{z n} \underline{z}_{0}\right) \cdot \underline{J}(\omega) e^{j \beta_{n}(\omega) z} d V}{2 \int_{S_{2}} \underline{e}_{t n} \times \underline{h}_{t n} \cdot \underline{z}_{0} d S_{2}}, \quad \text { (A2) } \begin{array}{l}
\text { in the structure with the hypothesis } \\
\text { ficient } c_{n}^{+}, \text {for a backward wave, } \\
\text { form: }
\end{array} \\
c_{n}^{+}\left(\omega, z_{1}, z_{2}\right) \cong-\frac{q}{4 \prod_{n}^{\prime}(\omega)} \int_{z_{1}}^{z_{2}} e_{z n}\left[\omega,\left.\underline{r}\left(z^{\prime}\right)\right|_{\text {part.traj. }}\right] e^{-j \omega\left(z^{\prime} / c\right)} e^{j \beta_{n}(\omega) z^{\prime}} d z^{\prime},
\end{gathered}
$$

where the sign "-" refers to the case of forward waves while the sign "+" to the backward ones.

The density current, in the time domain, of a particle of charge $q$ that moves along the waveguide (Fig. 5) at the speed of light, is given by

$$
\underline{J}(t)=q \underline{s}_{0}(s) \delta\left(t-\frac{s}{c}\right) \delta\left(x^{\prime}\right) \delta\left(y^{\prime}\right)
$$

where $s$ is the distance along the particle trajectory, $s_{0}$ is the unit vector tangent to the trajectory, and $\left(x^{\prime}, y^{\prime}\right)$ is the reference system on the plane normal to $\underline{s}_{0}$. In the frequency domain the expression (A3) becomes

$$
\underline{J}(\omega)=q \underline{s}_{0}(s) e^{-j \omega(s / c)} \delta\left(x^{\prime}\right) \delta\left(y^{\prime}\right) .
$$

Considering the beam loading $90^{\circ}$ out of phase in $\mathrm{rf}$ deflectors, one has to consider, in the scalar product $\underline{E} \cdot \underline{J}$ of Eq. (A2), only the longitudinal component of the electric field and density current. For a particle moving in the structure with the hypothesis $\underline{J} \cong \underline{J} \cdot z_{0}$, the coefficient $c_{n}^{+}$, for a backward wave, can be written in the

where $\left.\underline{r}\left(z^{\prime}\right)\right|_{\text {part.traj. }}$ is the transverse position of the particle along the structure (function of the longitudinal position $z^{\prime}$ ) and $\Pi_{n}^{\prime}$ is given by

$$
\Pi_{n}^{\prime}(\omega)=\frac{1}{2} \int_{S_{1}} \underline{e}_{t n} \times \underline{h}_{t n} \cdot \underline{z}_{0} d S_{1}
$$

It is easy to verify, from the analytical formulas [10], that

$$
\Pi_{n}(\omega)=\frac{1}{2} \int_{S_{1}} \underline{e}_{t n} \times \underline{h}_{t n}^{*} \cdot \underline{z}_{0} d S_{1}=-\Pi_{n}^{\prime}(\omega)
$$

where $\Pi_{n}$ is the power flow along the structure.

If the deflector length is $L$ and the particle enters at $z=0$ one obtains the following expression:

$$
c_{n}^{+}(\omega, z)=\frac{q}{4 \Pi_{n}(\omega)} \int_{z}^{L} e_{z n}\left[\omega,\left.\underline{r}\left(z^{\prime}\right)\right|_{\text {part.traj. }}\right] e^{-j \omega\left(z^{\prime} / c\right)} e^{j \beta_{n}(\omega) z^{\prime}} d z^{\prime}
$$

and, therefore Eq. (4).

\section{APPENDIX B: CALCULATION OF THE COEFFICIENT $c^{+}$IN THE CASE OF DISPERSION CURVE LINEARIZATION IN A LIMITED RANGE OF FREQUENCY [EQ. (6)]}

Since the major contribution to the integral (5) comes from a small range of frequencies near $f^{*}$ one can consider in such interval $e_{z}(\omega) \cong e_{z}\left(\omega^{*}\right)$ and one can develop to the first order in $\omega$ the exponential term obtaining

$$
-\omega \frac{z^{\prime}}{c}=-\omega^{*} \frac{z^{\prime}}{c}+\left(\omega^{*}-\omega\right) \frac{z^{\prime}}{c}, \quad \beta(\omega) z^{\prime} \cong \beta\left(\omega^{*}\right) z^{\prime}+\left.\frac{d \beta}{d \omega}\right|_{\omega=\omega^{*}}\left(\omega-\omega^{*}\right) z^{\prime} .
$$

Remembering that $\omega^{*} / c=\beta\left(\omega^{*}\right)$ and substituting (B1) in Eq. (4) one obtains

$$
c^{+}(\omega, z)=\frac{q}{4 \Pi\left(\omega^{*}\right)} \begin{cases}\left.\int_{z}^{L} e_{z}\left(\omega^{*}\right)\right|_{\text {part.traj. }} e^{j\left[\left.(d \beta / d \omega)\right|_{\omega=\omega^{*}}-(1 / c)\right]\left(\omega-\omega^{*}\right) z^{\prime}} & \left.d z^{\prime} \cong \int_{z}^{L} e_{z}\left(\omega^{*}\right)\right|_{\text {part.traj. }} e^{j\left[\left.(d \beta / d \omega)\right|_{\omega=\omega^{*}}\right]\left(\omega-\omega^{*}\right) z^{\prime}} d z^{\prime}, \\ \omega \in\left[\omega^{*}-\frac{\Delta \omega}{2}, \omega^{*}+\frac{\Delta \omega}{2}\right], & \text { elsewhere. }\end{cases}
$$


The approximation in the interval $\left[\omega^{*}-\Delta \omega / 2, \omega^{*}+\Delta \omega / 2\right]$ comes from the fact that the group velocity $v_{g}[=$ $\left.(d \beta / d \omega)^{-1}\right]$, for this kind of structures, is a few percent of the velocity of light.

Similarly the field given by the expression (5) can be developed to the first order the exponential term $-j \beta(\omega) z$ obtaining

$$
\begin{aligned}
\underline{E}^{+}(t, z) & \cong \frac{q}{4 \pi \Pi\left(\omega^{*}\right)} \operatorname{Re}\left[\underline{e}^{+}\left(\omega^{*}\right) \int_{z}^{L} e_{z}\left[\omega^{*},\left.\underline{r}\left(z^{\prime}\right)\right|_{\text {part.traj. }}\right] \int_{0}^{\infty} e^{\left.j(d \beta / d \omega)\right|_{\omega=\omega^{*}\left(\omega-\omega^{*}\right) z^{\prime}}} e^{-j \beta\left(\omega^{*}\right) z-\left.j(d \beta / d \omega)\right|_{\omega=\omega^{*}\left(\omega-\omega^{*}\right) z}} e^{j \omega t} d \omega d z^{\prime}\right] \\
& =\frac{q}{4 \pi \Pi\left(\omega^{*}\right)} \operatorname{Re}\left[\underline{e}^{+}\left(\omega^{*}\right) \int_{z}^{L} e_{z}\left[\omega^{*},\left.\underline{r}\left(z^{\prime}\right)\right|_{\text {part.traj. }}\right] e^{-\left.j(d \beta / d \omega)\right|_{\omega=\omega^{*}} \omega^{*}\left(z^{\prime}-z\right)-j \beta\left(\omega^{*}\right) z} \int_{0}^{\infty} e^{j \omega\left[t-\left.(d \beta / d \omega)\right|_{\omega=\omega^{*}}\left(z-z^{\prime}\right)\right]} d \omega d z^{\prime}\right] .
\end{aligned}
$$

Considering the integration in $\omega$ between $\omega^{*}-\Delta \omega / 2$ and $\omega^{*}+\Delta \omega / 2$ one obtains

$$
\int_{\omega^{*}-(\Delta \omega / 2)}^{\omega^{*}+(\Delta \omega / 2)} e^{j \omega\left[t-\left.(d \beta / d \omega)\right|_{\omega=\omega^{*}}\left(z-z^{\prime}\right)\right]} d \omega=e^{j \omega^{*}\left[t-\left.(d \beta / d \omega)\right|_{\omega=\omega^{*}}\left(z-z^{\prime}\right)\right]} \Delta \omega \operatorname{sinc}\left[\left(t-\frac{z-z^{\prime}}{v_{g}}\right) \frac{\Delta \omega}{2}\right],
$$

where $\operatorname{sinc}(x)=\sin (x) / x$.

\section{APPENDIX C: INTUITIVE APPROACH FOR THE WAKE FIELD CALCULATION IN A TW RF DEFLECTOR}

Considering the field configuration of Eq. (1) with a local excitation proportional to the leading charge displacement, the energy per unit length stored at the abscissa $z$ after the charge passage is given by

$$
U=\frac{1}{2} V(z) q=\frac{1}{2} \operatorname{Ekr}(z) q,
$$

where $-1 / 2 V(z)$ is the voltage seen by charge $q$ (the factor $1 / 2$ comes from the beam loading theorem), $r(z)$ is the $q$ displacement with respect to the axis, and $E / 2$ is the field amplitude.

Remembering the definition (11) of the $R / Q$ and that

$$
U=\frac{\Pi\left(\omega^{*}\right)}{v_{g}},
$$

one simply obtains the fact that the amplitude of the excited field at the abscissa $z$ is given by

$$
E=\frac{1}{2} k \omega^{*} \frac{R}{Q} r(z) q
$$

Since $v_{g} \ll v_{\mathrm{ph}}$ it is reasonable to suppose that the $E$ field generated by the particle passage has the rigid am- plitude profile given by (C3), a phase velocity equal to $c$, and a negative group velocity $v_{g}$ obtaining Eq. (12).

[1] CERN Report No. PS 2002-008 (RF), Geneve, 2002.

[2] J. Delahaye et al., in Proceedings of the EPAC, Stockholm, 1998 (Institute of Physics and IOP Publishing Ltd., London, 1998).

[3] H. Braun et al., CERN Report No. 99-06, Geneve, 1999.

[4] H. Braun, CERN Report No. CERN/PS/98-14 (LP), Geneve, 1998.

[5] C. Milardi et al., in Proceedings of the 2002 European Particle Accelerator Conference, Paris (EPS-IGA/ CERN, Geneva, 2002).

[6] D. Alesini et al., "The RF Deflectors for CTF3," CTFF3 Technical Note, INFN-LNF Accelerator Division, LNF Frascati, 2001.

[7] D. Alesini et al., in Proceedings of the 2002 European Particle Accelerator Conference, Paris (Ref. [5]).

[8] R. Corsini et al., CTF3 Note No. 054 (MD), Geneva, 2003.

[9] P. Bernard et al., CERN Report No. 68-30, 1968.

[10] Y. Garault, CERN Report No. 64-43, 1964.

[11] H. H. Braun et al., CERN Report No. 99-06, 1999.

[12] R. E. Collin, Foundation for Microwave Engineering (McGraw-Hill, New York, 1992).

[13] A. Gallo et al., in Proceedings of the European Particle Accelerator Conference, Vienna, 2000 (EPS-IGA/CERN, Geneva, 2000). 